\title{
Fermi-LAT constraints on dark matter annihilation cross section from observations of the Fornax cluster
}

\author{
Shin'ichiro Ando ${ }^{a, b}$ and Daisuke Nagai ${ }^{c, d, e}$ \\ ${ }^{a}$ Institute for Theoretical Physics, University of Amsterdam, 1090 GL Amsterdam, The \\ Netherlands \\ ${ }^{b}$ Gravitation and Astroparticle Physics, University of Amsterdam, 1090 GL Amsterdam, \\ The Netherlands \\ ${ }^{c}$ Department of Physics, Yale University, New Haven, CT 06520, USA \\ ${ }^{d}$ Department of Astronomy, Yale University, New Haven, CT 06520, USA \\ ${ }^{e}$ Yale Center for Astronomy and Astrophysics, Yale University, New Haven, CT 06520, USA \\ E-mail: s.ando@uva.nl, daisuke.nagai@yale.edu
}

\begin{abstract}
We analyze 2.8-yr data of $1-100 \mathrm{GeV}$ photons for clusters of galaxies, collected with the Large Area Telescope onboard the Fermi satellite. By analyzing 49 nearby massive clusters located at high Galactic latitudes, we find no excess gamma-ray emission towards directions of the galaxy clusters. Using flux upper limits, we show that the Fornax cluster provides the most stringent constraints on the dark matter annihilation cross section. Stacking a large sample of nearby clusters does not help improve the limit for most dark matter models. This suggests that a detailed modeling of the Fornax cluster is important for setting robust limits on the dark matter annihilation cross section based on clusters. We therefore perform the detailed mass modeling and predict the expected dark matter annihilation signals from the Fornax cluster, by taking into account effects of dark matter contraction and substructures. By modeling the mass distribution of baryons (stars and gas) around a central bright elliptical galaxy, NGC 1399, and using a modified contraction model motivated by numerical simulations, we show that the dark matter contraction boosts the annihilation signatures by a factor of 4 . For dark matter masses around $10 \mathrm{GeV}$, the upper limit obtained on the annihilation cross section times relative velocity is $\langle\sigma v\rangle \lesssim(2-3) \times 10^{-25} \mathrm{~cm}^{3} \mathrm{~s}^{-1}$, which is within a factor of 10 from the value required to explain the dark matter relic density. This effect is more robust than the annihilation boost due to substructure, and it is more important unless the mass of the smallest subhalos is much smaller than that of the Sun.
\end{abstract}

Keywords: dark matter theory, gamma ray theory, galaxy clusters

ArXiv ePrint: 1201.0753 


\section{Contents}

1 Introduction $\quad 1$

2 Gamma-ray intensity and dark matter density profiles $\quad 3$

2.1 Gamma-ray intensity from dark matter annihilation 3

2.2 Dark matter density profile: smooth component 3

2.3 Dark matter contraction due to baryon dissipation 5

$\begin{array}{lll}2.4 & \text { Effects of substructure } & 6\end{array}$

3 Constraints from a large statistical sample of clusters $\quad 6$

$\begin{array}{lll}3.1 & \text { Upper limits on individual clusters } & 6\end{array}$

$\begin{array}{lll}3.2 & \text { Stacking analysis } & 8\end{array}$

4 Constraints from the Fornax cluster $\quad \mathbf{1 0}$

$\begin{array}{lll}4.1 & \text { Distribution of dark matter, stars, and gas } & 10\end{array}$

4.2 Effects of dark matter contraction 11

$\begin{array}{lll}4.3 & \text { Effects of substructure boost and minimum mass } & 13\end{array}$

$\begin{array}{llr}5 & \text { Conclusions } & 16\end{array}$

$\begin{array}{lr}\text { A Analysis of Fermi-LAT data } & 17\end{array}$

$\begin{array}{lll}\text { A.1 Photon data processing } & 17\end{array}$

A.2 Maximum likelihood analysis 17

A.3 Setting upper limits on cluster emission 18

\section{Introduction}

Revealing the nature of dark matter is one of the important and challenging goals of modern physics, astrophysics, and cosmology. One of the avenues involves searching for annihilation signatures from dark matter halos in a form of gamma rays, neutrinos, and charged particles. Such a possibility is theoretically well motivated especially if dark matter is made of weakly interacting massive particles (WIMPs) such as the supersymmetric neutralino [1]. In addition, in order to thermally produce dark matter at the right abundance in the present Universe, WIMPs have to have some certain annihilation cross section (times relative velocity), most preferably, $\langle\sigma v\rangle=3 \times 10^{-26} \mathrm{~cm}^{3} \mathrm{~s}^{-1}[1-3]$. The recently launched Fermi Gamma-Ray Space Telescope and ground-based atmospheric Čerenkov telescopes such as HESS, MAGIC, and VERITAS have started to provide interesting constraints on the annihilation cross section of dark matter particles from various astrophysical objects, ranging from dwarf galaxies to the isotropic diffuse gamma-ray background, i.e., the emission coming from the entire Universe.

Clusters of galaxies are the largest concentrations of dark matter pulled together by gravity, and therefore, an attractive astrophysical object to look for the annihilation signals. The Fermi Large Area Telescope (LAT) collaboration analyzed their 11-month data to place constraints on the dark matter annihilation cross section [4] and decay [5] using observations of several nearby galaxy clusters. The obtained upper limits have already started to exclude 
some of the parameter space of WIMPs, and these cluster-based constraints are quite comparable to those obtained from dwarf galaxies [6-8] and the diffuse gamma-ray background [9].

In the previous analyses as well as theoretical studies on dark matter annihilation in galaxy clusters, it is commonly assumed that the dark matter density profile is smooth and characterized by the Navarro-Frenk-White (NFW) form [10]. However, it has been pointed out that the effects of subhalos might significantly boost the dark matter annihilation signal for massive clusters [11-13], if the smallest subhalo masses are of around Earth size as implied by arguments of the kinetic decoupling of WIMP particles in the early Universe [14-18]. In the case of the smooth NFW-like halos, the current upper limits on the cross section is about $\sim 30$ times larger than the canonical value $\left(\langle\sigma v\rangle=3 \times 10^{-26} \mathrm{~cm}^{3} \mathrm{~s}^{-1}\right)$ for the low-mass dark matter particles around $\sim 10 \mathrm{GeV}$. In the case of Earth-mass substructures, on the other hand, the canonical cross section has already been excluded for the low-mass WIMPs [4, 19], although there is a large uncertainty on the subhalo models, especially since it involves significant extrapolation of the subhalo mass functions down to small scales.

There is another effect that must be taken into account but has received little attention to date - the effect of dark matter contraction due to baryonic infall. Unlike dark matter, baryons can lose energy via radiative cooling and they fall towards the center of the halo, and as the result, modify gravitational potential in the central region. Dark matter is then dragged towards the center because of the deepened potential, modifying the density profile of dark matter [20-23]. Its implications for dark matter annihilation have been discussed in the context of galaxy-size halos [24, 25], but not in the context of cluster-size halos. Galaxy clusters contain a large amount of baryonic gas and stars, and in fact, the stars dominate the gravitational potential at central regions. Although there is no firm observational evidence for or against the halo contraction in massive clusters at the moment, the effect of the dark matter contraction is likely important for cluster-size halos.

In this paper, we analyze Fermi-LAT 2.8-yr data in order to constrain the dark matter annihilation signatures from galaxy clusters. As we shall show in this work, among several clusters analyzed thus far, the most stringent constraint on the annihilation cross section is obtained with Fornax, a nearby, well measured galaxy cluster located at $20 \mathrm{Mpc}$ away from Earth. We therefore focus on interpreting observations of the Fornax cluster, paying particular attention to modeling of dark matter contraction due to baryon dissipation as well as substructure boost, and address the relative importance of these two effects. Apart from its proximity and its large mass $\left(\sim 10^{14} h^{-1} M_{\odot}\right)$, there are several reasons why the Fornax cluster is ideal for detailed modeling. First, the Fornax cluster does not host any bright active galactic nuclei, unlike the Virgo and Perseus clusters. Secondly, it has regular thermal gas profiles and a spherical central massive elliptical galaxy, NGC 1399, and this feature makes the calculation of contraction based on the assumption of spherical symmetry quite reasonable. In order to infer the distribution of baryons, we use high-resolution observations of surface brightness profiles with Hubble Space Telescope (HST) for stars and with ROSAT $\mathrm{X}$-ray Telescope for thermal gas. Using the well-measured stellar and gas profiles, we then compute the dark matter density profile taking into account the dark matter contraction.

This paper is organized as follows. In Sec. 2, we present the formulation to compute intensities of gamma rays from dark matter annihilation, and models of dark matter density profiles, halo contraction due to baryon dissipation, and substructures from numerical simulations. In Sec. 3, we discuss the results of analysis for a large sample of galaxy clusters, including the upper limits on individual clusters and stacking analysis, highlighting that a detailed mass modeling of the Fornax cluster is indeed more important than the statistical 
constraint. Procedures for the data analysis of the Fermi-LAT data are summarized in Appendix A. In Sec. 4, we show results for the Fornax cluster, in which we present a detailed mass modeling of dark matter, stellar, and gaseous components in Fornax and discuss the relative importance of halo contraction and subhalo boost effects in modeling the annihilation signal from clusters. We finally conclude the present paper in Sec. 5. Throughout the paper, we assume the flat cold dark matter cosmology with cosmological constant $(\Lambda \mathrm{CDM})$, and adopt $\Omega_{m}=0.277, \Omega_{\Lambda}=0.723, H_{0}=100 h \mathrm{~km} \mathrm{~s}^{-1} \mathrm{Mpc}^{-1}$, and $h=0.7$.

\section{Gamma-ray intensity and dark matter density profiles}

In order to compute the gamma-ray intensity from dark matter annihilation, one needs to specify the dark matter annihilation mechanisms and the dark matter distribution in clusters. Here, after briefly introducing formalism to compute the intensity in Sec. 2.1, we discuss the density profiles obtained with numerical N-body simulations in the literature, where gravitational structure formation and evolution of only dark matter particles are followed. We introduce the smoothly distributed large-scale halo component (Sec. 2.2), effects of halo contraction due to baryon dissipation (Sec. 2.3), and small-scale substructure (Sec. 2.4).

\subsection{Gamma-ray intensity from dark matter annihilation}

The gamma-ray intensity, here defined as the number of photons received per unit area, time, solid angle, and energy, i.e., $I_{\gamma} \equiv d N_{\gamma} / d A d t d \Omega d E$, from annihilation of dark matter particles $\chi$, in a halo is given by

$$
I_{\gamma}(E, \theta)=\left.\frac{\langle\sigma v\rangle}{8 \pi(1+z)^{2} m_{\chi}^{2}} \frac{d N_{\gamma, \text { ann }}}{d E^{\prime}}\right|_{E^{\prime}=(1+z) E} \int d s \rho_{\chi}^{2}[r(s, \theta)],
$$

where $\theta$ is the angle from the halo center, $z$ is the redshift of the halo, $m_{\chi}$ is dark matter mass, and $d N_{\gamma, \text { ann }} / d E$ is the gamma-ray spectrum per annihilation. The intensity is proportional to the line-of-sight $(s)$ integral of dark matter density squared, $\rho_{\chi}^{2}$. Here, the density is assumed to be spherically symmetric, and the radius $r$ is related to $s$ and $\theta$ through $r=\sqrt{s^{2}+d_{A}^{2} \theta^{2}}$, where $d_{A}$ is the angular diameter distance to the halo.

The gamma-ray spectrum depends on the final state of annihilation. In this study, we adopt three different annihilation channels, $\chi \chi \rightarrow b \bar{b}, W^{+} W^{-}$, and $\tau^{+} \tau^{-}$. Figure 1 shows $E^{2} d N_{\gamma, \text { ann }} / d E$ for various dark matter masses between $10 \mathrm{GeV}$ and $1 \mathrm{TeV}$. The annihilation spectra for neutralino WIMPs are typically given by some combination of these three spectra. Note that there is no annihilation happening if the final state is $W^{+} W^{-}$and dark matter mass is smaller than $W^{ \pm}$mass $(80.4 \mathrm{GeV})$.

\subsection{Dark matter density profile: smooth component}

For the smooth component of the dark matter density profile, $\rho(r)$, we consider two models: one is the NFW profile [10], and the other is the Einasto profile with a central core [26, 27]. The NFW profile is given by

$$
\rho(r)=\frac{\rho_{s}}{\left(r / r_{s}\right)\left(r / r_{s}+1\right)^{2}},
$$

where $\rho_{s}$ and $r_{s}$ are the scale density and scale radius, respectively. The virial mass $M_{\text {vir }}$ and redshift $z$ are given inputs, and the virial radius $r_{\mathrm{vir}}$ is obtained by solving $M_{\mathrm{vir}}=$ $4 \pi r_{\text {vir }}^{3} \Delta_{\text {vir }}(z) \rho_{c}(z) / 3$, where $\rho_{c}(z)$ is the critical density, $\Delta_{\text {vir }}(z)=18 \pi^{2}+82 d-39 d^{2}$, and 


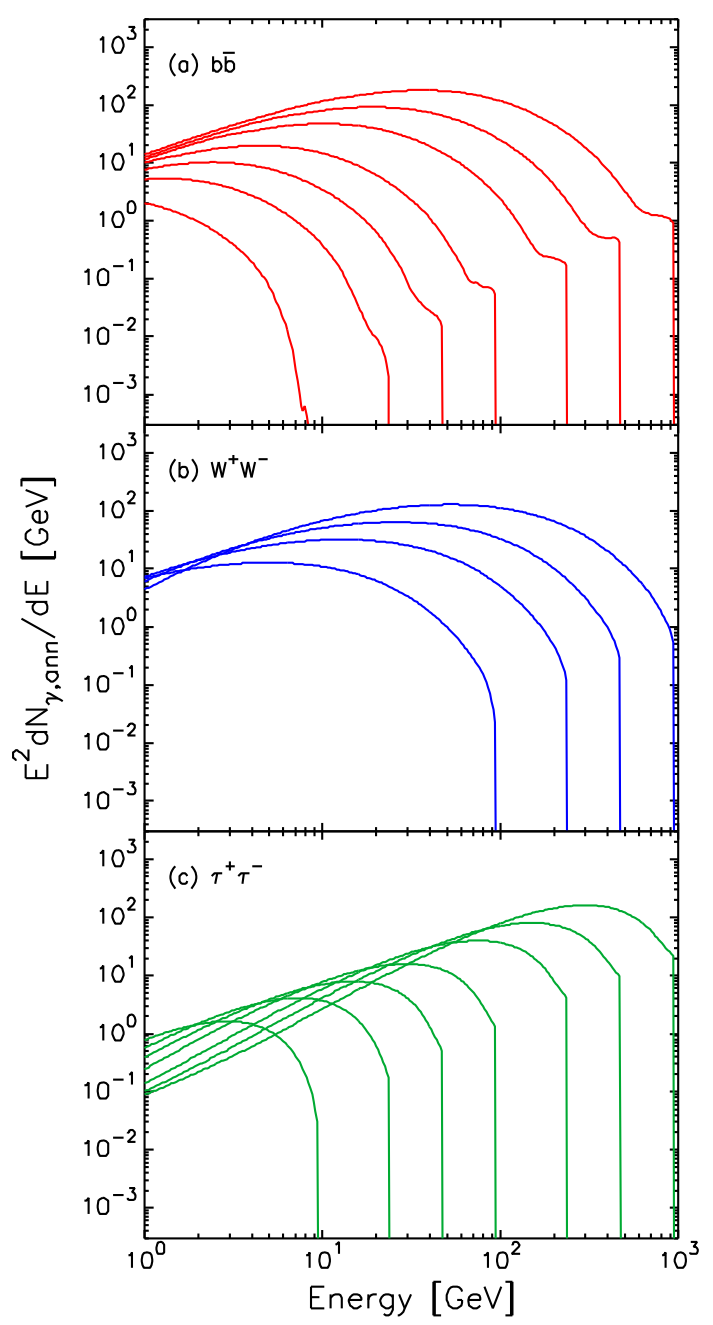

Figure 1. Gamma-ray spectrum per dark matter annihilation, $E^{2} d N_{\gamma, \text { ann }} / d E$ for (a) $b \bar{b}$, (b) $W^{+} W^{-}$, (c) $\tau^{+} \tau^{-}$annihilation channels. Assumed dark matter masses are $m_{\chi}=10,25,50,100,250,500$, and $1000 \mathrm{GeV}$ for the panels (a) and (c), and the same unless $m_{\chi}<m_{W}=80.4 \mathrm{GeV}$ for the panel (b).

$d=\Omega_{m}(1+z)^{3} /\left[\Omega_{m}(1+z)^{3}+\Omega_{\Lambda}\right]-1$ [28]. The scale radius $r_{s}$ is then defined as $r_{s}=r_{\mathrm{vir}} / c_{\mathrm{vir}}$, where $c_{\text {vir }}$ is the concentration parameter. We adopt the result of the mass-concentration relations from Ref. [29] (see also Refs. [30-33]):

$$
c_{\mathrm{vir}}\left(M_{\mathrm{vir}}, z\right)=\frac{7.85}{(1+z)^{0.71}}\left(\frac{M_{\mathrm{vir}}}{2 \times 10^{12} h^{-1} M_{\odot}}\right)^{-0.081} .
$$

By taking volume integral of $\rho(r)$ up to the virial radius $r_{\text {vir }}$ and then equating it to $M_{\text {vir }}$, we obtain

$$
\rho_{s}=\frac{M_{\mathrm{vir}}}{4 \pi r_{s}^{3}}\left[\ln \left(1+c_{\mathrm{vir}}\right)-\frac{c_{\mathrm{vir}}}{1+c_{\mathrm{vir}}}\right]^{-1}
$$


The Einasto profile, on the other hand, has the following functional form:

$$
\rho(r)=\rho_{e} \exp \left\{-d_{n}\left[\left(\frac{r}{r_{e}}\right)^{1 / n}-1\right]\right\},
$$

where $n$ and $r_{e}$ are the free parameters, and $d_{n}=3 n-1 / 3+0.0079 / n$. The normalization $\rho_{e}$ is obtained such that the total mass within $r_{\text {vir }}$ agrees with the measured $M_{\text {vir }}$ :

$$
\rho_{e}=\frac{M_{\mathrm{vir}} d_{n}^{3 n}}{4 \pi r_{e}^{3} n e^{d_{n}}} \Gamma\left(3 n, d_{n}\left[\frac{r_{\mathrm{vir}}}{r_{e}}\right]^{1 / n}\right)^{-1},
$$

where $\Gamma(a, x)$ is the lower incomplete gamma function.

Note that we also use other definitions of mass, including $M_{\Delta}$ with $\Delta=200$ or 500 , defined as the enclosed mass within a sphere with radius $r_{\Delta}$, within which the average density is $\Delta$ times the critical density of the universe. Conversion between different mass definitions is straightforward if one assumes density profile such as NFW as well as the concentration-mass relation (e.g., [34]).

\subsection{Dark matter contraction due to baryon dissipation}

The dark matter density profile is modified as a result of baryon dissipation. A so-called dark matter contraction model is based on the conservation of the angular momentum:

$$
M_{i}\left(\bar{r}_{i}\right) r_{i}=\left[M_{\mathrm{dm}, i}\left(\bar{r}_{i}\right)+M_{b, f}\left(\bar{r}_{f}\right)\right] r_{f},
$$

where $M(r)$ is the mass enclosed within the radius $r$, subscripts $\mathrm{dm}$ and $b$ are for dark matter and baryons, respectively, and subscripts $i$ and $f$ represent quantities before and after the contraction, respectively. The masses inferred from, e.g., gravitational lensing or Xray observations are the total mass, and therefore one must assume underlying distributions of both baryons and dark matter in order to infer $M_{\mathrm{dm}, i}(r)$. Here, when we discuss the initial density profile (before contraction), we simply assume that dark matter and baryons trace each other exactly; namely, $\rho_{\mathrm{dm}, i}(r)=\left(1-f_{b}\right) \rho_{i}(r)$ and $M_{\mathrm{dm}, i}(r)=\left(1-f_{b}\right) M_{i}(r)$, where $f_{b}$ is the baryon fraction at virial radius. For the final profiles of baryons, on the other hand, we use the observed distribution of stars and gas (as discussed in the later section), giving the baryon mass distribution to be $M_{b, f}(r) \equiv M_{\star}(r)+M_{\text {gas }}(r)$. Finally, $\bar{r}$ is the orbitaveraged radius for a particle found at $r$ in a simulation [22]. Equation (2.7) is solved for $r_{f}$, a contracted radius, as a function of the initial radius $r_{i}$. The dark matter mass profile after the contraction is then obtained by $M_{\mathrm{dm}, f}\left(r_{f}\right)=M_{\mathrm{dm}, i}\left(r_{i}\left(r_{f}\right)\right)$.

If particle orbits can be approximated to be circular and if the contraction happens adiabatically, then we simply have $\bar{r}=r$. This is a so-called standard adiabatic contraction model originally proposed in Ref. [20]. Reference [22], based on hydrodynamical simulations, presented the modified formula (2.7) and pointed out that corrections to the adiabatic contraction model can be accommodated by modifying a relation between $\bar{r}$ and $r$ as $\bar{r} / r_{\text {vir }}=0.85\left(r / r_{\text {vir }}\right)^{0.8}$. This was further investigated by various groups both theoretically $[25,35-41]$ and observationally [42-45]. Recently, Ref. [23] systematically studied this effect, and found that the relation

$$
\frac{\bar{r}}{r_{0}}=A_{0}\left(\frac{r}{r_{0}}\right)^{w}
$$


well represents the simulation results, where $r_{0}=0.03 r_{\text {vir }}$, and $w$ ranges from 0.5 to 1.3 , if $A_{0}$ is fixed to 1.6. Here we adopt $w=0.8$ as a canonical value for this parameter, but also consider the cases of $w=0.6$ and 1 . In addition, we adopt $A_{0}=1$ and $w=$ 1, the standard adiabatic contraction, as an extreme case scenario. Equation (2.7) has been calibrated only down to $r \sim 10^{-3} r_{\text {vir }}$ because of the resolution limit [23], but we shall extrapolate this relation further to very small radii. Although we assume angular momentum conservation, there is no guarantee that Eq. (2.8) remains valid below the resolution limit of the simulations. We therefore adopt yet another relation as a conservative scenario of the contraction: $\bar{r}=\max \left[A_{0} r_{0}\left(r / r_{0}\right)^{w}, 10^{-2} r_{\text {vir }}\right]$, which does not allow $\bar{r}$ to reach below $10^{-2} r_{\text {vir }}$, the current resolution limit of simulations. Note that in this model the magnitude of contraction is smaller for the larger $\bar{r}$. We discuss the application of this model to the Fornax cluster in Sec. 4.

\subsection{Effects of substructure}

If galaxy clusters consist of numerous subhalos as implied by numerical simulations, then the gamma-ray radiation may be dominated by the contribution from subhalos, even though they make up a small fraction of the total cluster mass. Here, we consider the results of recent numerical simulations of cluster-size halos that contain subhalos of $M_{\text {sub }}>5 \times 10^{7} M_{\odot}$, and its extrapolation down to Earth mass, $M_{\min }=10^{-6} M_{\odot}$, or even smaller mass $10^{-12} M_{\odot}$ [12]. (This is because the minimum mass of subhalos for neutralino dark matter models ranges widely, $M_{\min }=10^{-12}-10^{-4} M_{\odot}[18]$.) According to this model, the surface brightness profile contributed by subhalos are given by

$$
\begin{aligned}
I_{\gamma, \mathrm{sub}}(E, \theta) & =\frac{b\left(M_{200}\right) F_{\gamma, \mathrm{host}}(E)}{\pi \ln (17)} \frac{1}{\theta^{2}+\left(\theta_{200} / 4\right)^{2}}, \\
b\left(M_{200}\right) & =1.6 \times 10^{-3}\left(\frac{M_{200}}{M_{\odot}}\right)^{0.39},
\end{aligned}
$$

for $\theta \leq \theta_{200}$ and for $M_{\min }=10^{-6} M_{\odot}$. Here $\theta_{200} \equiv r_{200} / d_{A}$, the flux $F_{\gamma}$ is obtained by integrating $I_{\gamma}$ [Eq. (2.1)] over the solid angle, $b=F_{\gamma, \text { sub }} / F_{\gamma \text {,host }}$ is the boost factor, and the subscripts "sub" and "host" indicate subhalos and the host halo, respectively. Note that the boost factor decreases significantly for the larger values of minimum subhalo mass $M_{\min }$, and it scales roughly as $b \propto M_{\min }^{-0.2}[12]$.

\section{Constraints from a large statistical sample of clusters}

We discuss constraints on annihilation cross sections from 49 nearby rich galaxy clusters. After computing the intensity $I_{\gamma}(E, \theta)$ with Eq. (2.1) for these clusters, we analyze the Fermi-LAT data for 2.8 years to place constraints on $\langle\sigma v\rangle$. Unlike the previous study where clusters were assumed to be point-like sources [4], here we take the extension of the cluster emission fully into account. Analysis details can be found in the Appendix A. In this section, we consider only the NFW smooth halo model (Sec. 2.2) for a density profile, and other models are discussed in the following section.

\subsection{Upper limits on individual clusters}

We choose 23 galaxy clusters that are the brightest in X-rays from Ref. [46]. These are the same clusters as those analyzed in Ref. [47] for general gamma-ray analyses of galaxy clusters, 
Table 1. Parameters of galaxy clusters analyzed for dark matter annihilation. Upper sample is from Chandra Cluster Cosmology Project [48] and lower one is for the X-ray-selected bright clusters [46]. A2199 and A3571 are found in the both samples.

\begin{tabular}{|c|c|c|c|c|c|c|c|}
\hline Cluster & $l\left({ }^{\circ}\right)$ & $b\left({ }^{\circ}\right)$ & $z$ & $\begin{array}{c}M_{\text {vir }} \\
\left(10^{14} h^{-1} M_{\odot}\right)\end{array}$ & $\begin{array}{c}r_{\mathrm{vir}} \\
\left(h^{-1} \mathrm{Mpc}\right)\end{array}$ & $\begin{array}{l}F_{\lim }(>0.1 \mathrm{GeV}) \\
\left(10^{-9} \mathrm{~cm}^{-2} \mathrm{~s}^{-1}\right)\end{array}$ & $F_{\mathrm{DM}} / F_{\mathrm{lim}}$ \\
\hline A2142 & 44.23 & 48.69 & 0.0904 & 16.86 & 2.33 & 1.93 & 0.15 \\
\hline A3266 & 272.09 & -40.17 & 0.0602 & 12.57 & 2.15 & 2.23 & 0.23 \\
\hline A2029 & 6.50 & 50.55 & 0.0779 & 12.05 & 2.10 & 4.39 & 0.07 \\
\hline A401 & 164.18 & -38.87 & 0.0743 & 12.04 & 2.10 & 1.42 & 0.22 \\
\hline A754 & 239.25 & 24.76 & 0.0542 & 11.81 & 2.12 & 4.43 & 0.13 \\
\hline A 478 & 182.41 & -28.30 & 0.0881 & 11.35 & 2.05 & 2.06 & 0.10 \\
\hline A2256 & 111.10 & 31.74 & 0.0581 & 10.90 & 2.06 & 0.63 & 0.77 \\
\hline A3667 & 340.85 & -33.40 & 0.0557 & 10.20 & 2.01 & 4.56 & 0.11 \\
\hline A399 & 164.37 & -39.47 & 0.0713 & 8.53 & 1.88 & 3.83 & 0.07 \\
\hline A 85 & 115.05 & -72.06 & 0.0557 & 8.24 & 1.87 & 2.53 & 0.16 \\
\hline A3571 & 316.32 & 28.55 & 0.0386 & 8.13 & 1.88 & 1.19 & 0.72 \\
\hline ZwCl1215 & 282.50 & 65.19 & 0.0767 & 7.92 & 1.83 & 1.14 & 0.18 \\
\hline A1795 & 33.79 & 77.16 & 0.0622 & 7.51 & 1.81 & 1.01 & 0.30 \\
\hline A2065 & 42.88 & 56.56 & 0.0723 & 6.83 & 1.74 & 1.91 & 0.11 \\
\hline A3558 & 311.98 & 30.74 & 0.0469 & 6.54 & 1.74 & 0.94 & 0.51 \\
\hline A119 & 125.70 & -64.10 & 0.0445 & 6.15 & 1.71 & 2.57 & 0.20 \\
\hline A1644 & 304.91 & 45.50 & 0.0475 & 5.74 & 1.67 & 0.90 & 0.46 \\
\hline A3158 & 265.07 & -48.97 & 0.0583 & 5.63 & 1.65 & 1.97 & 0.14 \\
\hline A3391 & 262.36 & -25.16 & 0.0551 & 5.53 & 1.64 & 3.55 & 0.08 \\
\hline A3562 & 313.31 & 30.35 & 0.0489 & 4.44 & 1.53 & 0.76 & 0.41 \\
\hline A 2147 & 28.81 & 44.49 & 0.0355 & 4.19 & 1.51 & 3.25 & 0.18 \\
\hline A3376 & 246.53 & -26.29 & 0.0455 & 4.06 & 1.49 & 1.61 & 0.21 \\
\hline A496 & 209.59 & -36.49 & 0.0328 & 3.99 & 1.49 & 4.63 & 0.14 \\
\hline HydraA & 242.93 & 25.09 & 0.0549 & 3.81 & 1.45 & 3.23 & 0.07 \\
\hline A4059 & 356.83 & -76.06 & 0.0491 & 3.79 & 1.45 & 2.39 & 0.11 \\
\hline A2199 & 62.90 & 43.70 & 0.0304 & 3.73 & 1.46 & 2.99 & 0.24 \\
\hline A133 & 149.76 & -84.23 & 0.0569 & 3.45 & 1.40 & 1.74 & 0.11 \\
\hline A576 & 161.42 & 26.24 & 0.0401 & 3.14 & 1.37 & 2.81 & 0.12 \\
\hline $2 \mathrm{~A} 0335$ & 176.25 & -35.08 & 0.0346 & 3.12 & 1.37 & 2.56 & 0.18 \\
\hline A2657 & 96.65 & -50.30 & 0.0402 & 3.00 & 1.35 & 2.54 & 0.13 \\
\hline A2063 & 12.85 & 49.71 & 0.0342 & 2.96 & 1.35 & 1.85 & 0.25 \\
\hline A1736 & 312.58 & 35.10 & 0.0449 & 2.81 & 1.32 & 3.14 & 0.08 \\
\hline MKW3s & 11.39 & 49.45 & 0.0453 & 2.79 & 1.31 & 2.08 & 0.12 \\
\hline A2589 & 94.66 & -41.20 & 0.0411 & 2.59 & 1.28 & 1.30 & 0.21 \\
\hline A2052 & 9.39 & 50.10 & 0.0345 & 2.45 & 1.27 & 1.95 & 0.19 \\
\hline A2634 & 103.45 & -33.06 & 0.0305 & 2.31 & 1.24 & 2.62 & 0.18 \\
\hline A4038 & 24.89 & -75.82 & 0.0288 & 2.19 & 1.22 & 2.67 & 0.19 \\
\hline EXO0422 & 203.30 & -36.16 & 0.0382 & 2.00 & 1.18 & 4.67 & 0.06 \\
\hline $\mathrm{A} 0754$ & 239.25 & 24.75 & 0.0528 & 13.57 & 2.22 & 4.43 & 0.16 \\
\hline A1367 & 234.80 & 73.03 & 0.0216 & 7.08 & 1.82 & 4.61 & 0.53 \\
\hline Centaurus & 302.41 & 21.56 & 0.0499 & 3.19 & 1.37 & 2.93 & 0.08 \\
\hline Coma & 58.09 & 87.96 & 0.0232 & 9.60 & 2.01 & 2.79 & 1.00 \\
\hline Fornax & 236.72 & -53.64 & 0.0046 & 1.17 & 1.01 & 0.54 & 21.51 \\
\hline Hydra & 269.63 & 26.51 & 0.0114 & 2.31 & 1.26 & 3.77 & 0.89 \\
\hline M49 & 286.92 & 70.17 & 0.0044 & 0.60 & 0.80 & 0.93 & 7.54 \\
\hline NGC4636 & 297.75 & 65.47 & 0.0037 & 0.16 & 0.51 & 1.25 & 2.48 \\
\hline NGC5044 & 311.23 & 46.10 & 0.0090 & 0.43 & 0.72 & 1.54 & 0.82 \\
\hline NGC5813 & 359.18 & 49.85 & 0.0064 & 0.38 & 0.69 & 3.06 & 0.73 \\
\hline NGC5846 & 0.43 & 48.80 & 0.0061 & 0.15 & 0.51 & 3.71 & 0.31 \\
\hline
\end{tabular}

where the sample clusters are chosen such that they yield the largest mass divided by distance squared, which is a reliable measure of expected annihilation fluxes. In addition, we also analyze 34 massive low-redshift clusters from the Chandra Cluster Cosmology Project [48]. We include this sample in order to investigate the level of cosmic-ray pressure in clusters and their implications for cluster-based cosmological tests [49], which we will discuss in more 


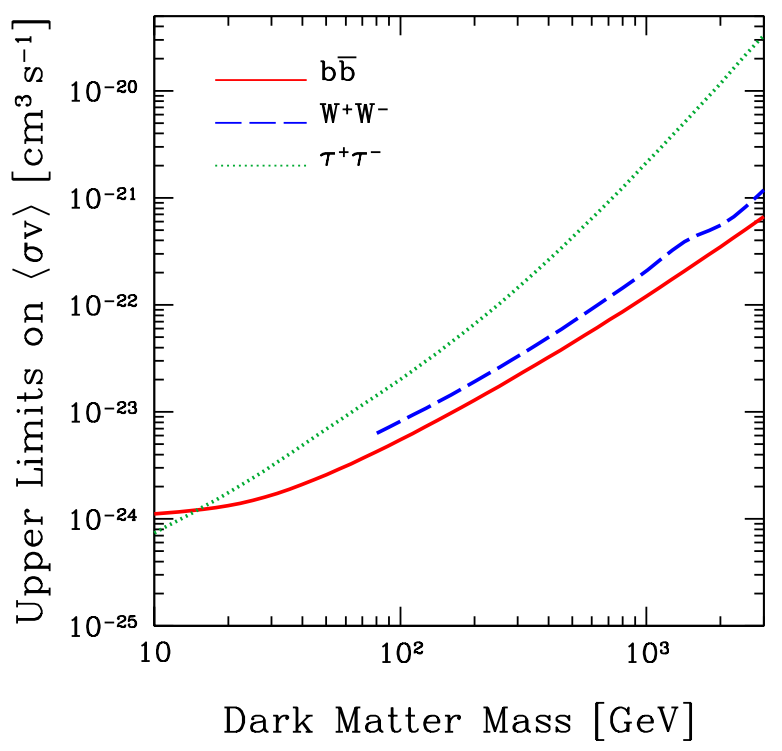

Figure 2. The cross section upper limits from Fornax cluster (smooth NFW with no contraction) for the $b \bar{b}, W^{+} W^{-}$, and $\tau^{+} \tau^{-}$annihilation channels.

detail in our subsequent work. We remove clusters located at low Galactic latitudes, $|b|<20^{\circ}$, in the analysis because of the strong emission from the Galactic plane. Consequently, we have 49 clusters to analyze. Two of them (A2199 and A3571) are found in both samples. Table 1 summarizes properties of these clusters.

In the table we also show the flux upper limits for each cluster assuming $E^{-2}$ spectrum and that the clusters are point-like. The seventh column shows the integrated flux upper limits in $0.1-100 \mathrm{GeV}, F_{\mathrm{lim}}(>0.1 \mathrm{GeV})$, and they are typically $(1-5) \times 10^{-9} \mathrm{~cm}^{-2} \mathrm{~s}^{-1}$. The last column of the table shows the ratio of expected gamma-ray flux from dark matter annihilation in the case of smooth NFW halo model to the flux upper limits, with respect to the value for the Coma cluster. These ratios, therefore, indicate the promise of each cluster as a target for dark matter annihilation. It is clear from this table that the Fornax cluster is by far the most promising target among 49 clusters considered in this work.

In Fig. 2, we show cross section upper limits as a function of WIMP mass for the three different annihilation channels obtained from observations of the Fornax cluster. The channel into gauge bosons will give similar yield to $b \bar{b}$ for all the masses, but limits for $\tau$-lepton pairs are significantly weaker especially at larger masses, because of hard spectrum (Fig. 1). We note that these limits are consistent with those of Refs. [4, 19] after correcting for differences in exposures.

\subsection{Stacking analysis}

One may wonder if stacking a large statistical sample of galaxy clusters improves upper limits on the annihilation cross section from those obtained individually. Here, we stack a subset of 49 galaxy clusters discussed above, which is a significant improvement from the previous studies based on 6 or 8 clusters $[4,19]$. The procedure for obtaining the stacking constraint on the cross section is similar to the individual cluster analysis (see Appendix A for more 


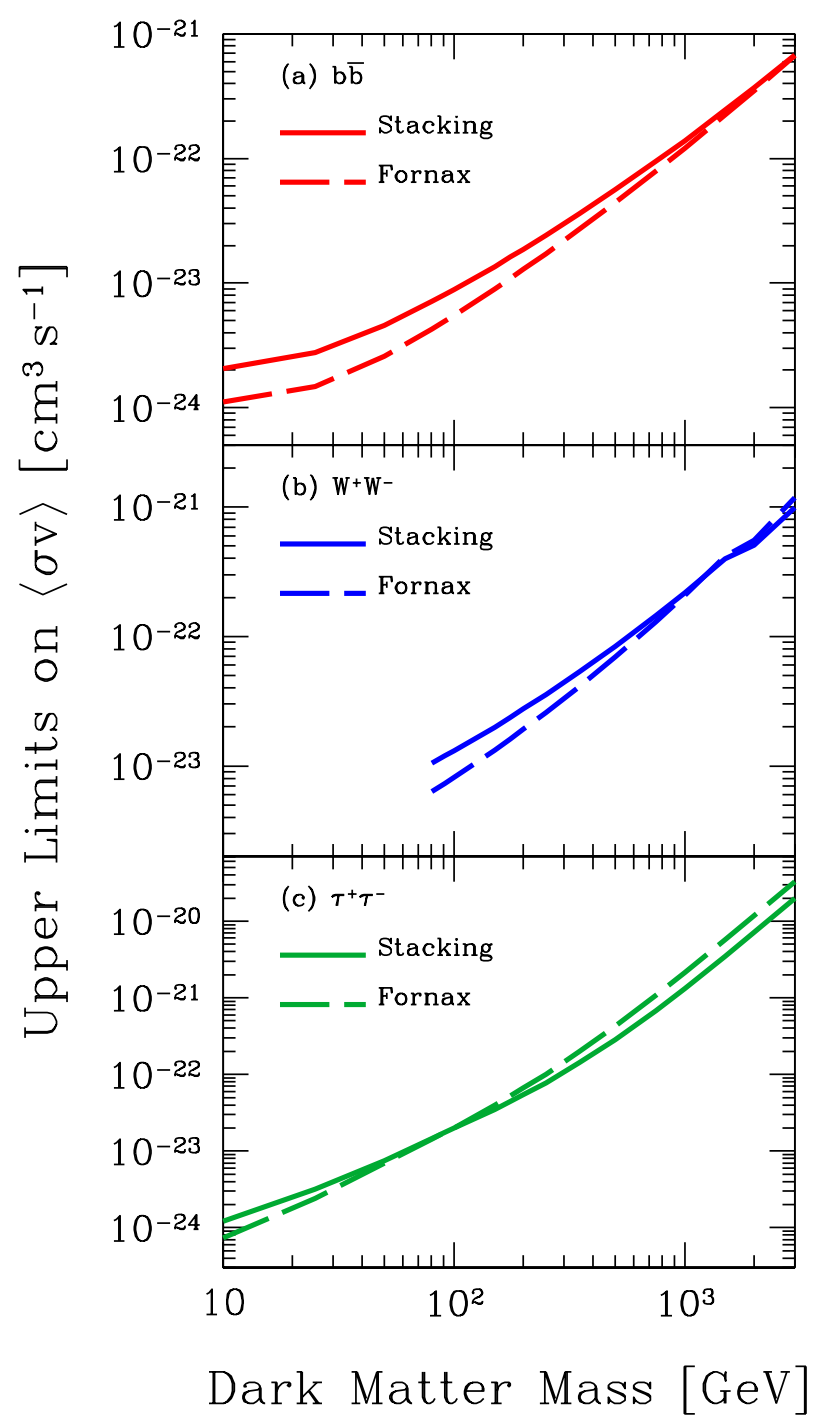

Figure 3. Upper limits on annihilation cross section $\langle\sigma v\rangle$ for the $b \bar{b}, W^{+} W^{-}$, and $\tau^{+} \tau^{-}$annihilation channels, in the case of smooth NFW host halo model (with no contraction). Solid and dashed curves are the limits due to staked clusters and Fornax, respectively.

details). In order not to bias the limits too high, we remove clusters with signal-to-noise ratio larger than $3 \sigma$ compared with the (fixed) background model for any dark matter masses and annihilation channels. ${ }^{1}$ As the result, we have 38 clusters used in the stacking analysis.

In Fig. 3, we compare the cross section upper limits from the stacking analysis and the constraint from the Fornax cluster alone for the three different annihilation channels. We find that the stacked limits of the cross section degrade toward lower dark matter mass, while they improve by about a factor of 2 at multi-TeV mass regime for the $\tau^{+} \tau^{-}$channel.

\footnotetext{
${ }^{1}$ Note that this does not mean that some clusters are already detected with $\gtrsim 3 \sigma$, because there are uncertainties in our procedure of fixing background.
} 
This is in part because we still have weak (less than $3 \sigma$ ) excess emission from some galaxy clusters for some annihilation models. This shows that the Fornax cluster dominates the constraint on the annihilation cross section, and stacking a large sample of clusters does not help improve the limits. This is in contrast to the case of dwarf satellite galaxies, where the stacking analysis of 10 satellite galaxies can improve the limits by a factor of a few for a wide range of dark matter masses $[7,8]$. In the next section, we shall discuss that treating the Fornax cluster carefully is indeed more important for setting an improved constraint on the annihilation cross section based on galaxy clusters.

\section{Constraints from the Fornax cluster}

As shown in Table 1, the Fornax cluster is located at $(l, b)=\left(236.72^{\circ},-53.64^{\circ}\right)$ in the Galactic coordinate. Its redshift is $z=0.0046$, and the corresponding angular diameter distance is $d_{A}=13.7 h^{-1} \mathrm{Mpc}$. Both optical and X-ray observations show that the brightness profiles of Fornax are fairly regular, and therefore, modeling dark matter distribution assuming spherical symmetry is well justified.

\subsection{Distribution of dark matter, stars, and gas}

The X-ray brightness has been used to infer the mass to be $M_{500}=6.4 \times 10^{13} h^{-1} M_{\odot}[46,50]$ (see also Refs. [51, 52]), and the corresponding virial mass and concentration are $M_{\text {vir }}=$ $1.2 \times 10^{14} h^{-1} M_{\odot}$ and $c_{\mathrm{vir}}=5.6$, respectively. For the Einasto profile, Eq. (2.5), we adopt $n=3.87$ and $r_{e}=1247 \mathrm{kpc}$, following the cluster simulation of similar size (model C09 of Ref. [26]).

We shall obtain $M_{\star}(r)$ and $M_{\text {gas }}(r)$ as they are necessary ingredients for calculating the final dark matter profile after contraction using Eq. (2.7). For the stellar mass distribution, we use the HST observation of the bright elliptical galaxy, NGC 1399, located at the center of the Fornax cluster. The surface brightness profile of stars are well represented by the "Nuker law" [53]:

$$
I(\theta)=2^{(\beta-\gamma) / \alpha} I_{b}\left(\frac{\theta}{\theta_{b}}\right)^{-\gamma}\left[1+\left(\frac{\theta}{\theta_{b}}\right)^{\alpha}\right]^{(\gamma-\beta) / \alpha},
$$

which behaves as $\theta^{-\gamma}\left(\theta^{-\beta}\right)$ at small (large) $\theta$ with a break around $\theta_{b}$. We here consider the results of HST WFPC2 observations using the F606W filter $[54,55]: \alpha=1.58, \beta=1.63$, $\gamma=0.09, \theta_{b}=3.17^{\prime \prime}$, and $I_{b}=17.23 \mathrm{mag} \operatorname{arcsec}^{-2}$. This photometry result is valid down to a very small radii, $\sim 1 \mathrm{pc}$ (i.e., $\sim 0.01^{\prime \prime}$ ). The same measurements also constrain the ellipticity of the galaxy, which is found very small [54]. Given that the assumption of spherical symmetry is well justified, we obtain the luminosity density by de-projection [56]:

$$
j(r)=-4 \int_{r / d_{A}}^{\infty} \frac{I^{\prime}(\theta) d \theta}{\sqrt{\theta^{2} d_{A}^{2}-r^{2}}} .
$$

In order to obtain the density profile, we finally multiply $j(r)$ by the mass-to-light ratio, $\rho_{\star}(r)=(M / L) j(r)$. According to the stellar kinematics study, it is believed that the gravitational potential in the central regions is dominated by stellar component, and the mass-tolight ratio has been inferred to be about 10 times that of the Sun $[57,58]$, which is typical for old stellar population. Figure 4 shows the density profile of the stellar component, $\rho_{\star}$, obtained in this way, and it dominates the mass within $\sim 10 \mathrm{kpc}$. We note that the power-law 

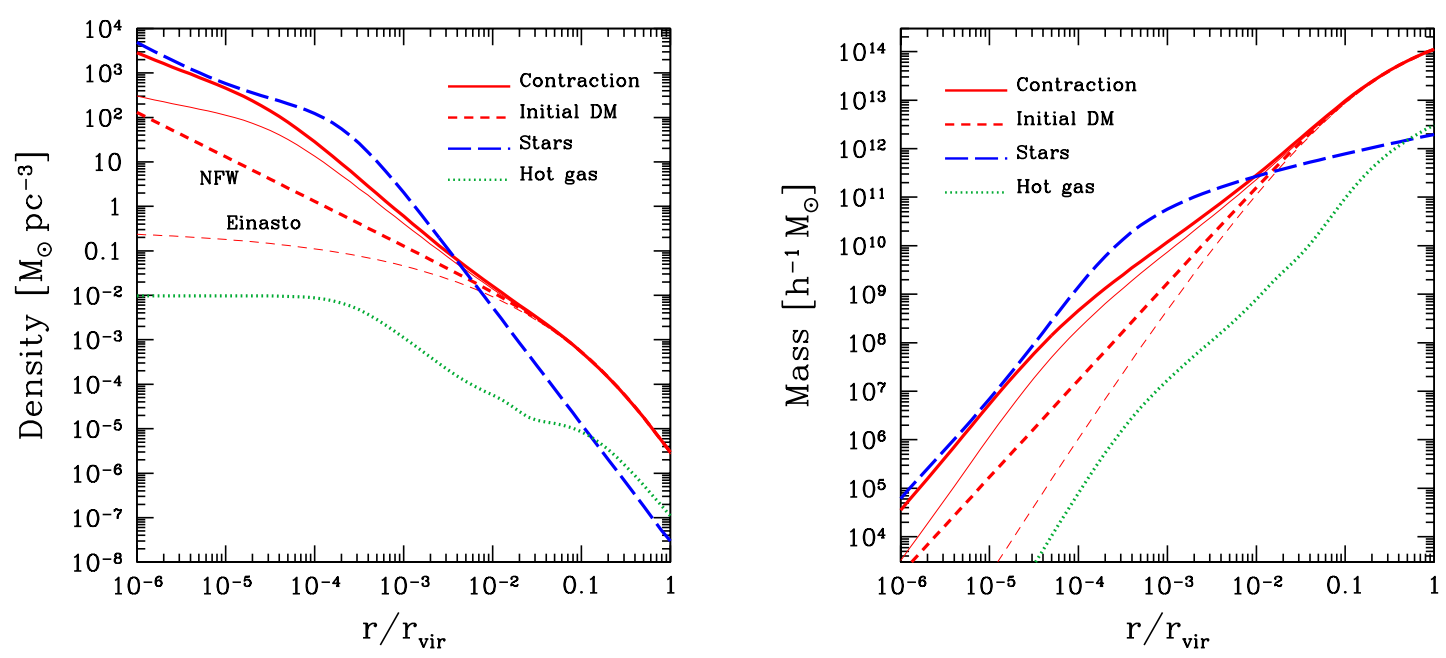

Figure 4. Density (left) and mass (right) profiles of dark matter (before contraction in short-dashed line; after contraction in solid line), stars (long-dashed), and baryonic gas (dotted). For dark matter before and after contraction, both the NFW (thick) and Einasto (thin) profiles are shown. The canonical parameters $\left(A_{0}=1.6\right.$ and $\left.w=0.8\right)$ are adopted for the contraction model.

extrapolation of Eq. (4.1) works reasonably well even at large radii, but underestimates the brightness (and therefore gives conservative results) beyond $\sim 20 \mathrm{kpc}[59]$.

The thermal gas component can be inferred from X-ray data. Here we use the ROSAT observations of the Fornax cluster [60,61]. In particular, Ref. [61] identified three different components and fitted each with a bi-dimensional $\beta$-profile. The thermal gas density and mass profile are shown in Figure 4. They are subdominant compared to the stellar density and mass within $\sim 100 \mathrm{kpc}$, and hence do not play important role in the dark matter contraction directly. However, since it is the dominant baryon component around the virial radius, it affects the dark matter contraction calculation through $f_{b}$ in an indirect way.

\subsection{Effects of dark matter contraction}

In Fig. 4, we show both initial and final dark matter density profiles for the Fornax cluster. We consider the NFW and Einasto models as the initial dark matter profile for the Fornax cluster and adopt the canonical contraction model $\left(A_{0}=1.6\right.$ and $\left.w=0.8\right)$. The initial dark matter profiles are in good agreement with each other at radii larger than $\sim 10 \mathrm{kpc}$, beyond which the total mass has been measured. Although the dark matter density is still subdominant at the smaller radii compared to the baryon density dominated by stars, it is enhanced significantly because of the dark matter contraction due to baryonic infall. This is the case even if the original profile has an inner core as the Einasto profile.

Once the density profile is obtained, we compute the gamma-ray intensity using Eq. (2.1). Figure 5 shows the intensity integrated over energy, $I_{\gamma}(\theta)$, for both the NFW and Einasto profiles as initial density models. Here, we fix particle-physics parameters to be $N_{\gamma \text {,ann }}\langle\sigma v\rangle / m_{\chi}^{2}=$ $10^{-26} \mathrm{~cm}^{3} \mathrm{~s}^{-1} \mathrm{GeV}^{-2}$. In order to see what range of $\theta$ contributes to the total signals the most, we multiply $I_{\gamma}(\theta)$ by $\theta^{2}$, such that the area below each curve gives the gamma-ray flux. One notices that there are double-peak structures. One peak at higher $\theta$ corresponds to the scale radius $r_{s}$ of the NFW or Einasto profiles. The other peak, on the other hand, 


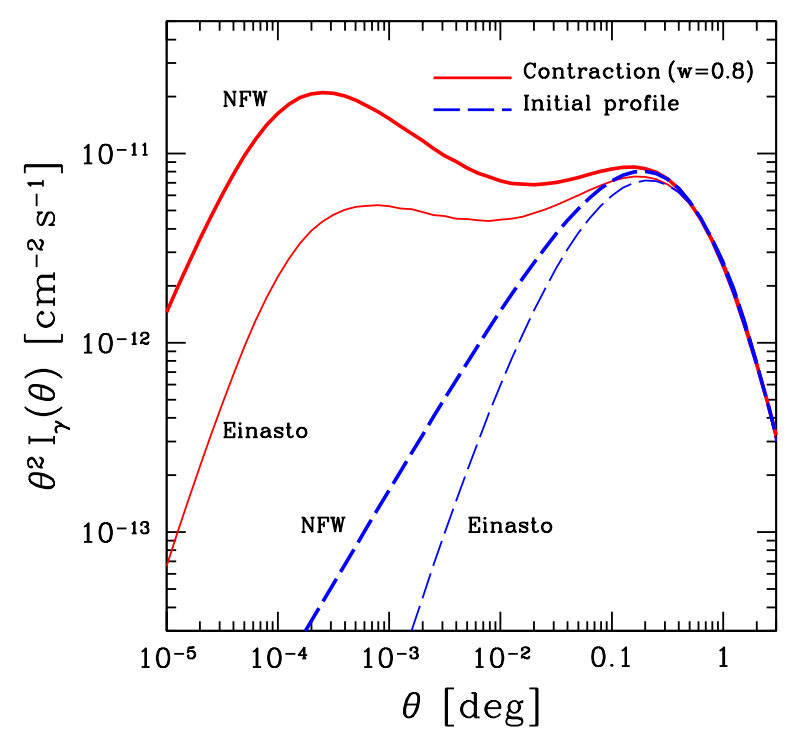

Figure 5. Gamma-ray intensity as a function of angle from the cluster center before and after the dark matter contraction, for both the NFW and Einasto profiles. Note that the instrumental responses have not been included yet. The particle-physics parameters are fixed to be $N_{\gamma, \text { ann }}\langle\sigma v\rangle / m_{\chi}^{2}=10^{-26} \mathrm{~cm}^{3} \mathrm{~s}^{-1} \mathrm{GeV}^{-2}$.

corresponds to the characteristic scale of the dark matter contraction. Since the typical angular resolutions of the current generation of gamma-ray telescopes are on the order of $0.1-1^{\circ}$ (depending on energies of gamma rays), the peaks at lower $\theta$ will simply make a central pixel brighter but do not make additional resolvable features in the maps.

The left panel of Fig. 6 shows a density enhancement factor after dark matter contraction, $\rho_{\mathrm{dm}, f} / \rho_{\mathrm{dm}, i}$, for various dark matter contraction models. Here we assume the NFW model as an initial density profile. We compare results for three different values of $w=0.6$, 0.8 , and 1 by fixing $A_{0}=1.6$ in Eq. (2.8). The larger the parameter $w$ is, the more efficient the contraction gets, since $\bar{r}$ is smaller. We also show results of two extreme scenarios. One is the standard adiabatic contraction model $\left(A_{0}=1\right.$ and $\left.w=1\right)$, and the other is the broken power-law model where $\bar{r}$ follows Eq. (2.8) with $w=0.6$ at large radii but becomes constant $\left(\bar{r}=10^{-2} r_{\text {vir }}\right)$ for small radii [see discussions after Eq. (2.8)]; they are labeled as "Adiabatic" and "Break," respectively. The true model is likely bracketed by these extreme cases.

The right panel of Fig. 6 shows the gamma-ray intensities for the various contraction models considered above. As shown in the left panel of Fig. 6, the flux enhancements are bracketed by the two extreme models. Although the peak moves towards larger $\theta$ for smaller $\bar{r}$, one cannot resolve such peak yet with the current gamma-ray telescopes. The inner peaks are around $10^{-5}-10^{-3} \mathrm{deg}$, which corresponds to $3-300 \mathrm{pc}$ in the physical size. By integrating over $\theta$ out to large values, we find that the gamma-ray flux is boosted by a factor of $2-7$ by the dark matter contraction effect.

We show, in Fig. 7, the upper limits (corresponding to $95 \%$ credible interval) on the annihilation cross section, $\langle\sigma v\rangle$, as a function of dark matter mass for the initial NFW and Einasto profiles, and for various annihilation channels. We compare no contraction model with the canonical contraction model $\left(A_{0}=1.6\right.$ and $\left.w=0.8\right)$. The contraction model 

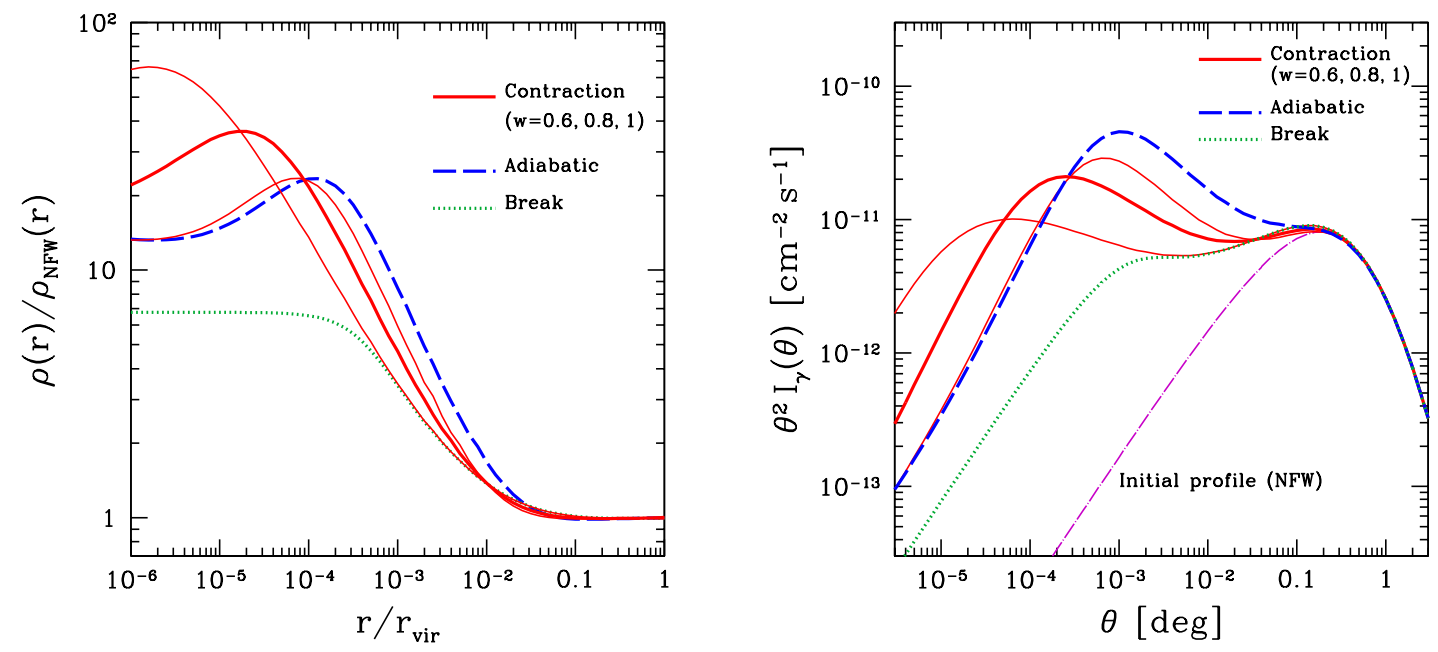

Figure 6. Density enhancement of dark matter, $\rho_{\chi}(r) / \rho_{\mathrm{NFW}}(r)$ (left) and gamma-ray intensity as a function of angle from the cluster center (right), due to the contraction for the initial NFW model. Solid curves are for the power-law models of $\bar{r}$ [Eq. (2.8)], with $w=0.6$ (top at low radii), 0.8 (middle), and 1 (bottom). Dashed line is for the standard adiabatic contraction model $\left(A_{0}=1, w=1\right)$, and dotted line is for $A_{0}=1.6, w=0.6$ but with a power-law break in Eq. (2.8) such that the $\bar{r}$ gets constant at $10^{-2} r_{\text {vir }}$ for small radii.

improves the limits at $m_{\chi}=100 \mathrm{GeV}$ by a factor of 4.1 for the $b \bar{b}$ and $W^{+} W^{-}$channels, and 3.8 for the $\tau^{+} \tau^{-}$channel in the case of the NFW initial profile, and this is almost independent of mass. In the case of the Einasto initial profile, on the other hand, the improvement factors at $100 \mathrm{GeV}$ are 2.4 for $b \bar{b}$ and $W^{+} W^{-}$, and 2.2 for $\tau^{+} \tau^{-}$. We note that the limits for nocontraction models of the NFW and Einasto profiles differ by only $\sim 20 \%$ (the former is better), which is negligible compared to the differences between models with and without contraction.

We compare various contraction models for the NFW initial profile and the $b \bar{b}$ annihilation channel in Fig. 8. As expected, the improvement factor is bracketed by our two extreme models, between 1.9 and 6.3 for the mass of $100 \mathrm{GeV}$ and the $b \bar{b}$ channel. It is worth pointing out that even the very conservative model of the contraction with the power-law break at small radii in Eq. (2.8) gives a factor of 2 enhancement compared to the no-contraction model, highlighting the importance of dark matter contraction in interpretation of the dark matter annihilation signals from galaxy clusters. This effect will make clusters brighter in a manner independent of the boost due to subhalos, which is the effect of much interest in the past several years and revisited below.

\subsection{Effects of substructure boost and minimum mass}

If the gamma rays are dominated by annihilation in subhalos and the subhalo mass function extends down to Earth mass (i.e., $M_{\min }=10^{-6} M_{\odot}$ ), then the cross section upper limits are significantly improved. Figure 9 shows the intensity $I_{\gamma}(\theta)$ integrated over energy, as a function of angle $\theta$, for the Fornax cluster. Here, we fix the particle-physics parameter to be $N_{\gamma, \text { ann }}\langle\sigma v\rangle / m_{\chi}^{2}=3 \times 10^{-30} \mathrm{~cm}^{3} \mathrm{~s}^{-1} \mathrm{GeV}^{-2}$. We show the contributions from both the smooth NFW host halo and substructures. For the latter, we adopt two minimum subhalo masses: $5 \times 10^{7} M_{\odot}$ corresponding to the current resolution limit [12] and $10^{-6} M_{\odot}$ corresponding to the 


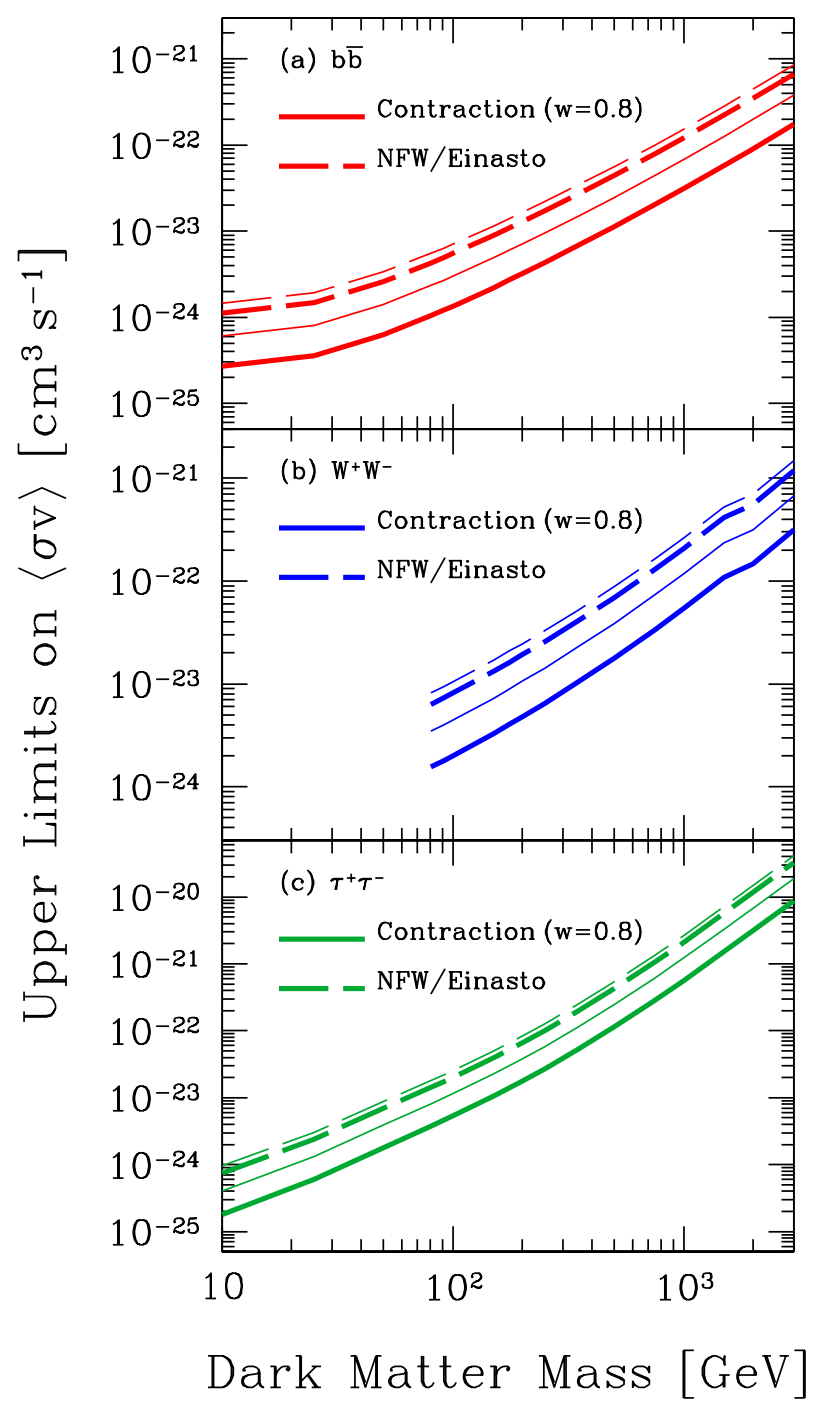

Figure 7. Upper limits (corresponding to $95 \%$ credible interval) on annihilation cross section $\langle\sigma v\rangle$ as a function of dark matter mass. The limits for the initial profiles (no-contraction model) are compared with those for the canonical contraction model with $A_{0}=1.6$ and $w=0.8$. Thick and thin curves correspond to the NFW and Einasto profiles, respectively.

typical smallest subhalo mass for the neutralino dark matter. One can see that the subhalo contribution could exceed that of the host halo if the subhalo mass function extends down to the Earth-mass scale. However, the relative importance of the host vs. subhalo contribution is highly sensitive to the cutoff mass scale. For example, if it is around the current resolution limit of simulations, then the host halo contribution becomes more dominant.

Next, we investigate the dependence of the upper limit of dark matter annihilation cross section from Fornax on the assumed minimum subhalo mass $M_{\min }$. Results are shown in Fig. 10 for $m_{\chi}=100 \mathrm{GeV}$ and the three different annihilation channels. Here we adopt the NFW profile with no contraction for the smooth component. Assuming the $b \propto M_{\min }^{-0.2}$ 


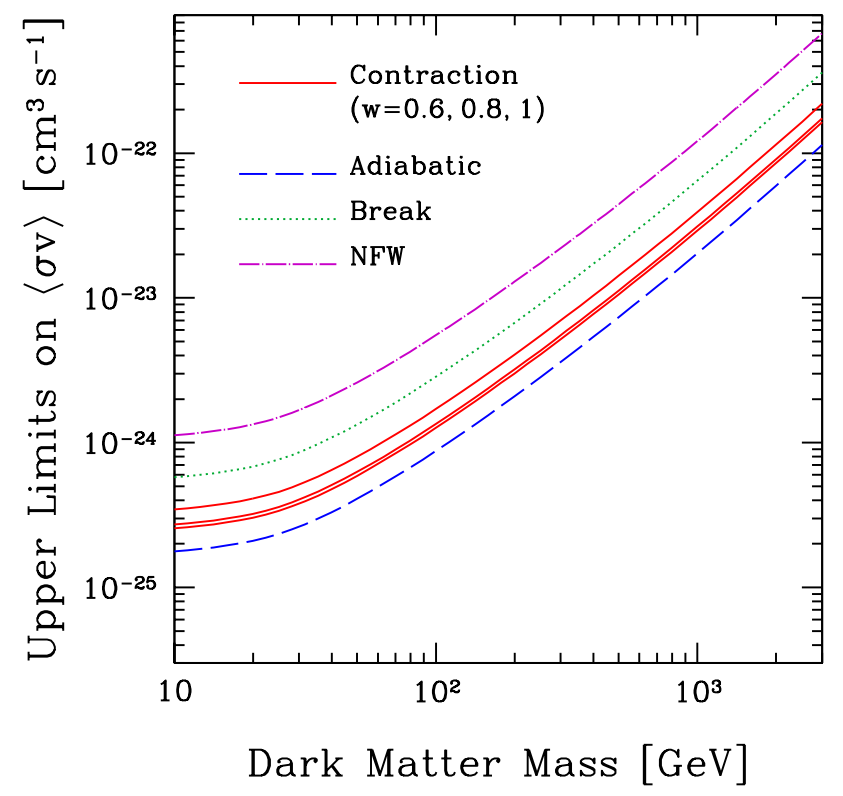

Figure 8. Cross section upper limits $\langle\sigma v\rangle$ for various contraction models for the NFW initial profile and the $b \bar{b}$ annihilation channel. The line types are the same as those in Fig. 6.

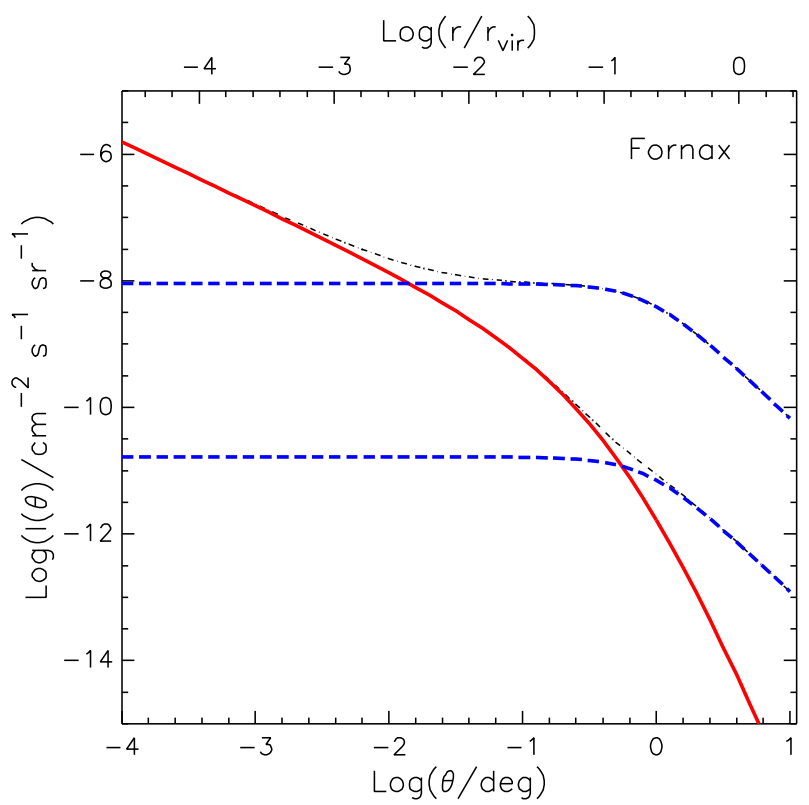

Figure 9. Intensity $I(\theta)$ as a function of angle $\theta$ (and radius in units of $r_{\text {vir }}$ ) for the Fornax cluster. Dark matter parameters are fixed to be $N_{\gamma, \text { ann }}\langle\sigma v\rangle / m_{\chi}^{2}=3 \times 10^{-30} \mathrm{~cm}^{3} \mathrm{~s}^{-1}$. Solid curve is the contribution from smooth NFW component, and dashed curves are those from subhalos assuming minimum subhalo mass of $M_{\min }=5 \times 10^{7} M_{\odot}$ (lower) and $M_{\min }=10^{-6} M_{\odot}$ (upper). Total intensities are shown as dot-dashed curves. 


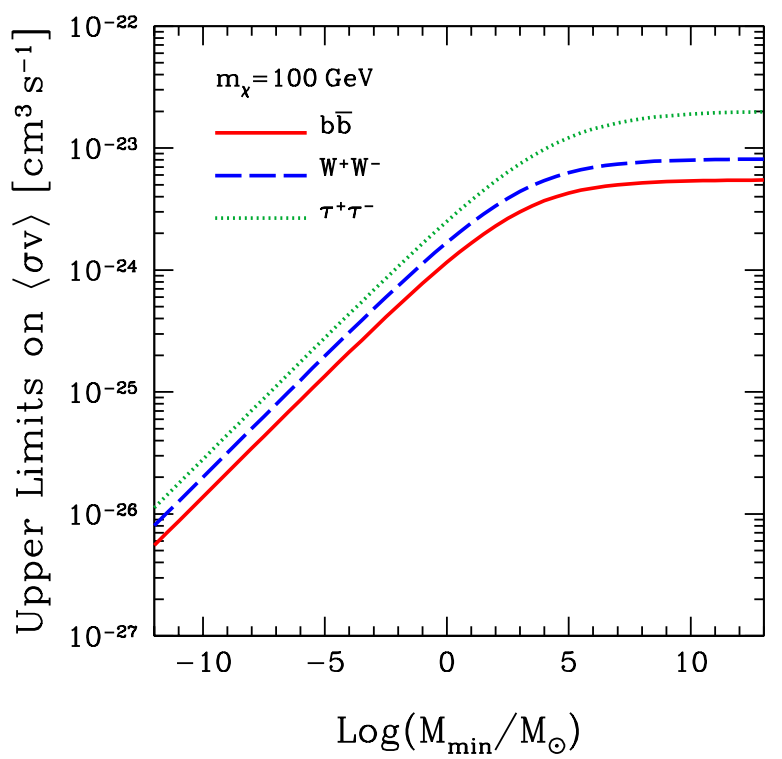

Figure 10. Upper limits on annihilation cross section $\langle\sigma v\rangle$, obtained with the Fornax cluster, as a function of minimum subhalo mass, $M_{\text {min }}$, for the three different annihilation channels and $m_{\chi}=$ $100 \mathrm{GeV}$. For the smooth component, the NFW profile with no contraction is adopted.

dependence, we find that the upper limits on $\langle\sigma v\rangle$ scales as $M_{\min }^{0.2}$, when $M_{\min }$ is small and the emission is dominated by annihilation in subhalos. This is realized for $M_{\min } \lesssim$ $10^{2} M_{\odot}$. For larger $M_{\min }$ than this value, the emission is dominated by the smooth NFW host-halo component and the limits asymptotically approach the values evaluated assuming no substructure. The canonical cross section of $3 \times 10^{-26} \mathrm{~cm}^{3} \mathrm{~s}^{-1}$ is excluded, only if $M_{\text {min }}$ is smaller than the Earth mass. We once again stress that the estimate of substructure boost factor (by Earth-size dark matter subhalos) requires extrapolation of the subhalo mass function by more than 13 orders of magnitude, given that the state-of-the-art numerical simulations can resolve halos of only $M \sim 5 \times 10^{7} M_{\odot}$ level [12].

Comparing the relative importance of substructure boost to the effect of dark matter contraction, we conclude that the substructure boost could exceed the contraction boost, only if the minimum subhalo mass is considerably smaller than one solar mass. Otherwise, the substructure boost is at most a factor of a few, and the effect is generally smaller than the effects of contraction that ranges between a factor of 2 and 6 . While the substructure boost remains highly uncertain, it is critical to take into account the contraction by baryon dissipation when studying the dark matter annihilation signals from clusters.

\section{Conclusions}

We investigated dark matter annihilation in galaxy clusters and constraints on annihilation cross sections with gamma-ray data from Fermi-LAT. Our main results are summarized below.

By analyzing 49 nearby massive galaxy clusters located at high Galactic latitudes, we show that the Fornax cluster provides the most stringent constraints on the dark matter annihilation cross section. In addition, we show that the stacking analysis does not help 
improve the cross section upper limits much. Our results suggest that detailed mass modeling of the Fornax cluster is more important for improving the dark matter annihilation cross section constraints from clusters than improving the cluster sample size.

We therefore performed a detailed mass modeling and predicted expected dark matter annihilation signals of the Fornax cluster, by taking into account effects of dark matter contraction and substructures. While the latter has been considered in the literature, the effect of dark matter contraction by baryon dissipation has not been considered in interpretation of the dark matter annihilation signal in cluster-size halos to date. By modeling the mass distribution of baryons (stars and gas) around a central bright elliptical galaxy, NGC 1399, and using a modified contraction model motivated by numerical simulations, we show that the dark matter contraction boosts annihilation signatures by a factor of $\sim 4$. Within a conservative range of contraction parameters, we also show that the flux boost will fall between a factor of 2 and 7 .

We analyzed the Fermi-LAT data for 2.8 years around the Fornax cluster. After taking all the sources and diffuse backgrounds into account, we obtained upper limits on the annihilation cross section for various models of contraction. We showed that the limits are improved, compared to those for the NFW or Einasto profiles with no contraction, by a factor of 2-6 for a wide range of parameters of the contraction model. For the canonical contraction model, the current limits are $\langle\sigma v\rangle \lesssim(2-3) \times 10^{-25} \mathrm{~cm}^{3} \mathrm{~s}^{-1}$ for dark matter with mass around $10 \mathrm{GeV}$, which is roughly one order of magnitude larger than the canonical cross section necessary to explain the thermal relic density. We argue that this effect is more robust than the subhalo boost that has been discussed in the literature, and indeed, more important unless the minimum subhalo mass is considerably smaller than the mass of the Sun.

\section{Acknowledgments}

We thank Eiichiro Komatsu for enlightening discussions, Roberto Trotta for helpful discussions, and Savvas Koushiappas for comments. SA was supported by the GRAPPA initiative at University of Amsterdam and in part by Japan Society for Promotion of Science. DN was supported in part by NSF grant AST-1009811, by NASA ATP grant NNX11AE07G, and by the Yale University.

\section{A Analysis of Fermi-LAT data}

\section{A.1 Photon data processing}

We perform the analysis using the Fermi Science Tools (v9r23p1), as well as data of gammaray photons collected by Fermi-LAT for the mission elapsed time (MET) between $239557417 \mathrm{~s}$ and 329159098 s (2.8 years; from August 4, 2008 to June 7, 2011), contained in a region of interest (ROI), i.e., a circle of $10^{\circ}$-radius around a target cluster. First, we chose DIFFUSE and DATACLEAN class photons whose energies are between $1 \mathrm{GeV}$ and $100 \mathrm{GeV}$ by using gtselect, such that contamination of charged particles gets reduced to minimum. Then with gtmktime we further cut the data according to the spacecraft configuration, using "DATA_QUAL==1 \&\& LAT_CONFIG==1 \&\& ABS (ROCK_ANGLE) $<52$ " filter as well as ROI-based zenith angle cut.

\section{A.2 Maximum likelihood analysis}

Using these filtered data around the cluster, we perform the binned likelihood analysis, by comparing the photon data with the models of sources (both point-like and extended), as 

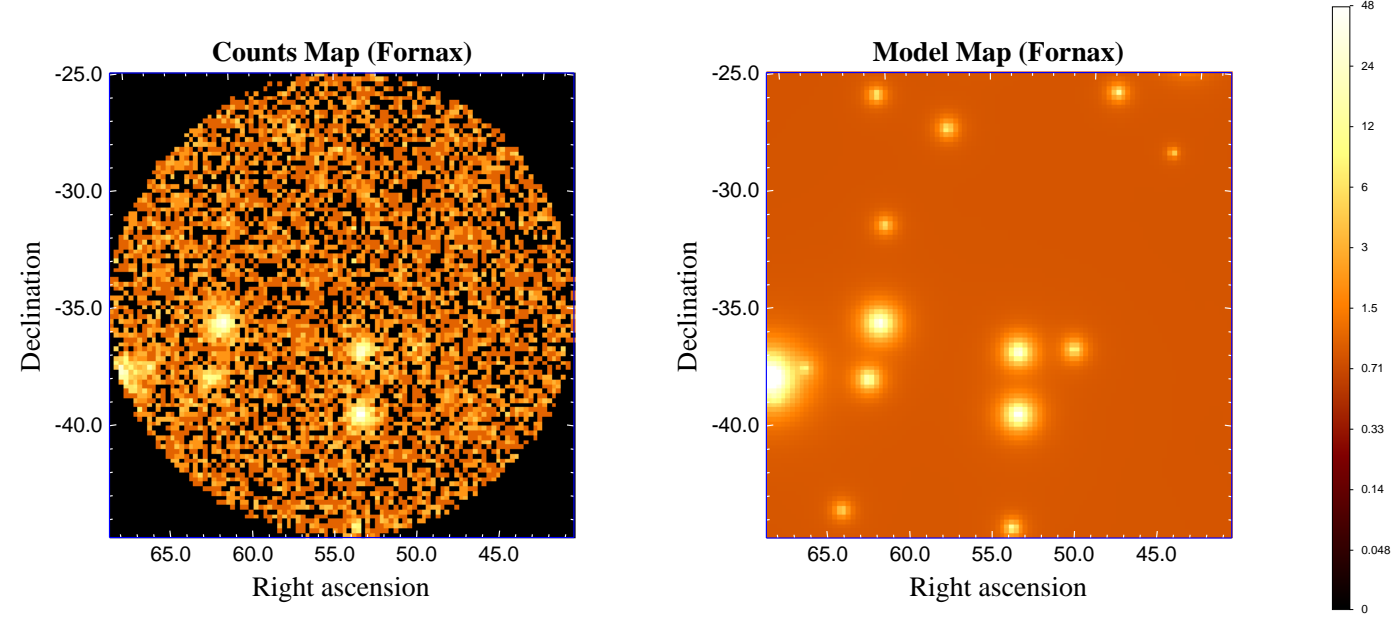

Figure 11. Counts map (left) and model map (right) for 1-100 GeV photons around the Fornax cluster. The colors show source counts per pixel and are logarithmically spaced. Ten 2FGL point sources are found in ROI ( $10^{\circ}$ radii) and 28 total are found in the source region $\left(15^{\circ}\right.$ radii), in addition to the both Galactic and extragalactic diffuse backgrounds. Note that the flux of the source at far left in the model back is saturated.

well as the Galactic foreground and extragalactic background. The sources are taken from 2-year source catalog (2FGL) [62], if they are located within $15^{\circ}$ radii (source region) from the cluster. With gtbin, we divide the cluster map into spatial $100 \times 100$ pixels, whose size is $0.2^{\circ} \times 0.2^{\circ}$, and also into 20 energy bins equally spaced logarithmically between $1 \mathrm{GeV}$ and $100 \mathrm{GeV}$. The left panel of Fig. 11 shows such a map (for Fornax) of photon counts per pixel, but integrated over the entire energy range, 1-100 GeV. Based on this binning, with gtltcube followed by gtexpcube2, we produce exposure maps corresponding to each cluster, and then with gtsrcmaps, maps of the sources and the diffuse backgrounds in ROI.

We use gtlike to perform the maximum likelihood analysis, first with DRMNFB then followed by NEWMINUIT optimizers. This way, we constrain amplitudes of both the Galactic foreground and the extragalactic background, and also for most of the sources, the flux and the power-law index of the spectra if they are within ROI. If the sources are out of ROI but still within source region, then the fixed values from 2FGL catalog for these parameters are used. Then finally, by combining results of likelihood analysis, we create the model maps with gtmodel, to be compared with the photon count maps. The right panel of Fig. 11 shows the best-fit model map of the data shown in the left panel.

\section{A.3 Setting upper limits on cluster emission}

To make templates of cluster emission, we convolve cluster brightness profiles with the P6_V11 instrumental response functions (IRFs) toward the direction of ROI. Since the size of the cluster (i.e., $\left.r_{s} / d_{A}\right)$ and the point spread function (PSF) is much smaller than ROI, we here use smaller regions $\left(\sim 2^{\circ} \times 2^{\circ}\right)$ with finer pixel sizes $\left(0.02^{\circ} \times 0.02^{\circ}\right)$ around clusters for obtaining the upper limits. For this new pixelization, we again run gtbin to make photon count maps such as the one shown in Fig. 12, and gtmodel to make model maps of the cluster as well as those of the backgrounds and the other sources. We show the model maps for the Fornax cluster after convolving with the IRFs in Fig. 13 for both smooth NFW host-halo 


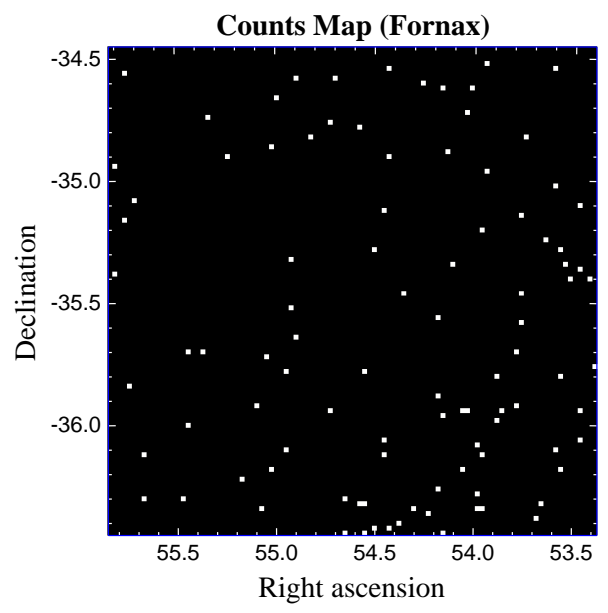

Figure 12. The same as the left panel of Fig. 11, but with finer pixelization. White dots represent single photons received.
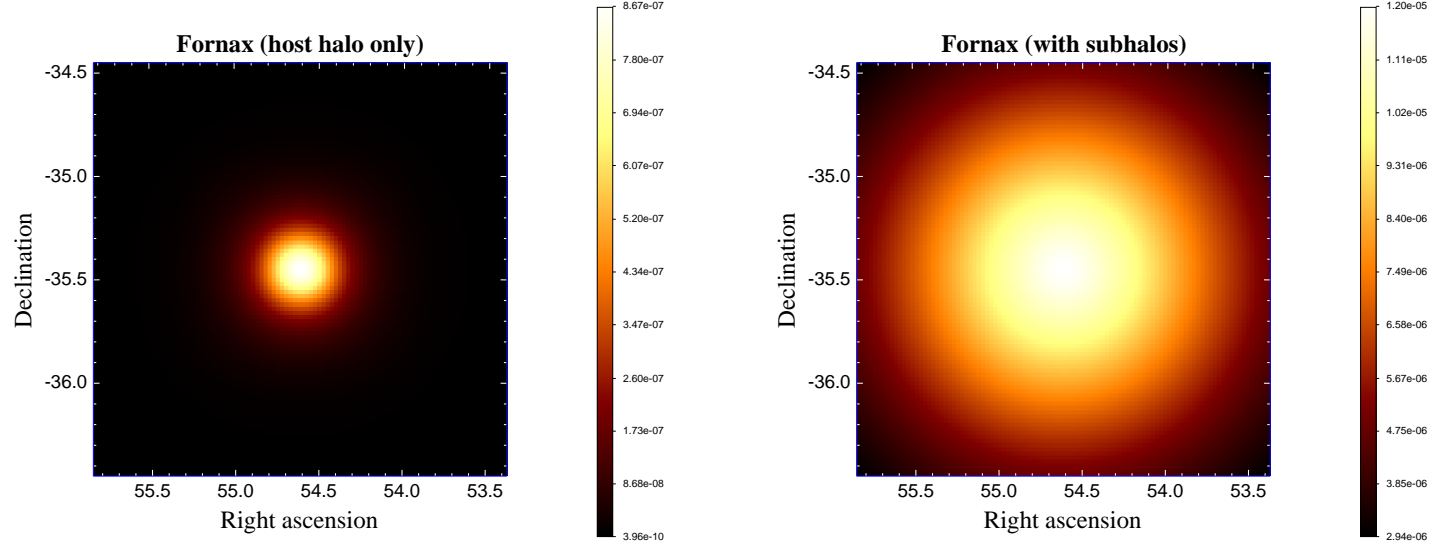

Figure 13. The gamma-ray model maps of Fornax for the NFW density profile (left) and host plus subhalo model (right). The colors represent expected photon counts per $0.02^{\circ} \times 0.02^{\circ}$ pixel (in linear scaling), where $b \bar{b}$ annihilation channel with $m_{\chi}=100 \mathrm{GeV}$ and $\langle\sigma v\rangle=10^{-26} \mathrm{~cm}^{3} \mathrm{~s}^{-1}$ is assumed, and photon energies are between $7.94 \mathrm{GeV}$ and $10 \mathrm{GeV}$. No backgrounds and other sources are included in these maps.

case and the case with large boost due to subhalos (with $M_{\min }=10^{-6} M_{\odot}$ ). One can see that the latter is much more extended than the former. Note, however, that the backgrounds and other sources are not included in these maps. Although we do not show the maps for the contracted cluster model, they look similar to the map for NFW.

We obtain upper limits on cluster emission by comparing the models with the data. According to the Bayesian statistics (e.g., [63]), the posterior probability distribution function for some theoretical parameter $\theta$ (in this case, the annihilation cross section, $\theta=\langle\sigma v\rangle$ ) given data $d$ is

$$
P(\theta \mid d) \propto P(\theta) L(d \mid \theta),
$$




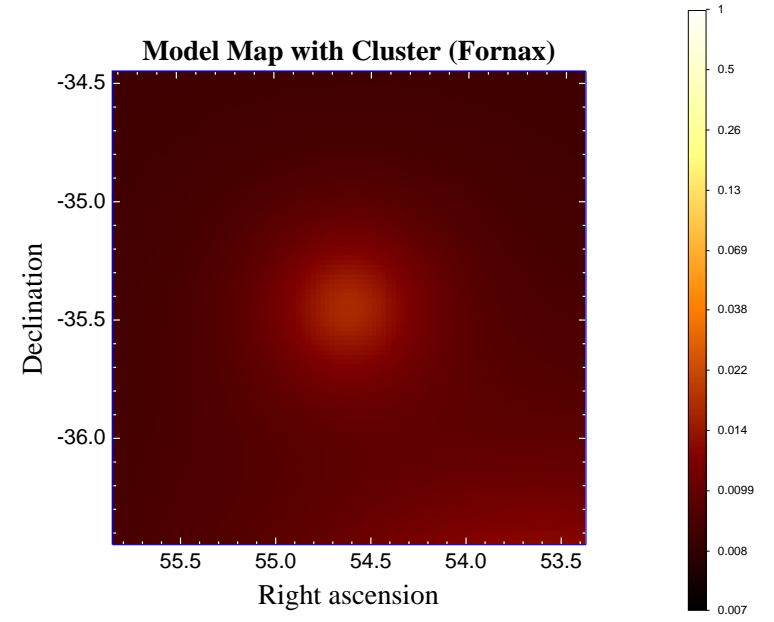

Figure 14. Model map of the Fornax cluster combined with the diffuse backgrounds as well as point sources, integrated for 1-100 GeV. This map is to be compared with the counts map of Fig. 12; the color scaling is set such that white represents single photon received per pixel, while black does less than 0.007 photon. The cluster dark matter component is from the smooth NFW halo, with $b \bar{b}$ annihilation channel, $m_{\chi}=100 \mathrm{GeV}$, and $\langle\sigma v\rangle=5.5 \times 10^{-24} \mathrm{~cm}^{3} \mathrm{~s}^{-1}$ that is the $95 \%$ credible upper limit from the current data.

where $P(\theta)$ is the prior distribution, for which we adopt the uniform and improper prior, $P(\theta)=$ const., for positive $\theta$ and zero otherwise, and $L(d \mid \theta)$ is the Poisson likelihood function. The latter is specifically given as

$$
L=\prod_{i=1}^{N_{\mathrm{pix}}} \frac{\mu_{i}^{d_{i}} e^{-\mu_{i}}}{d_{i} !},
$$

where subscript $i$ represents pixel number, $\mu_{i}$ and $d_{i}$ are theoretical model and the data counts in the pixel $i$, respectively. We assume that the model is divided into the signal $\left(s_{i}\right)$ and background $\left(b_{i}\right)$ contributions, $\mu_{i}=s_{i}+b_{i}$, where the former depends on the theoretical parameter $\theta$ and the latter includes both the diffuse backgrounds and 2FGL sources. We here fix all the parameters for $b_{i}$ to the values obtained with gtlike in the previous subsection, and then obtain an upper limit on $\theta$ by solving

$$
1-\alpha=\int_{0}^{\theta_{\lim }} d \theta P(\theta \mid d),
$$

for $\theta_{\lim }$. In particular, we are interested in upper limits corresponding to $95 \%$ credible interval, and for that we adopt $\alpha=0.05$ above. As an example, in Fig. 14, we show what the Fornax cluster map would appear when it is just as bright as currently allowed and is dominated by the smooth NFW component (with no contraction). The photon counts per $0.02^{\circ} \times 0.02^{\circ}$ pixel could be at most 0.017 at the center of the map.

When we obtain upper limits with stacked cluster maps, we generalize Eq. (A.2) to include contributions from different clusters as

$$
L=\prod_{c l, i} \frac{\mu_{c l, i}^{d_{c l, i}} e^{-\mu_{c l, i}}}{d_{c l, i} !},
$$


where the subscript $c l$ represents clusters. Here we remove some clusters from the stacking analysis, if they are brighter than $3 \sigma$ level compared with the backgrounds for any dark matter masses and the annihilation channels. For this purpose, we also use the posterior probability distribution function introduced above, $P(\theta \mid d)$, and by fixing the parameters for the background models $b_{i}$. As the result, we have 38 clusters used in the stacking analysis. A more careful treatment for the gamma-ray detection from the galaxy clusters should be made by varying background parameters together with $\theta$. However, this is beyond the scope of the present study and will be revisited in future publication.

\section{References}

[1] G. Jungman, M. Kamionkowski and K. Griest, "Supersymmetric dark matter," Phys. Rept. 267, 195 (1996) [hep-ph/9506380].

[2] L. Bergstrom, "Nonbaryonic dark matter: Observational evidence and detection methods," Rept. Prog. Phys. 63, 793 (2000) [hep-ph/0002126].

[3] G. Bertone, D. Hooper and J. Silk, "Particle dark matter: Evidence, candidates and constraints," Phys. Rept. 405, 279 (2005) [hep-ph/0404175].

[4] M. Ackermann et al., "Constraints on Dark Matter Annihilation in Clusters of Galaxies with the Fermi Large Area Telescope," JCAP 1005, 025 (2010) [arXiv:1002.2239 [astro-ph.CO]].

[5] L. Dugger, T. E. Jeltema and S. Profumo, "Constraints on Decaying Dark Matter from Fermi Observations of Nearby Galaxies and Clusters," JCAP 1012, 015 (2010) [arXiv:1009.5988 [astro-ph.HE]].

[6] A. A. Abdo et al., "Observations of Milky Way Dwarf Spheroidal galaxies with the Fermi-LAT detector and constraints on Dark Matter models," Astrophys. J. 712, 147 (2010) [arXiv:1001.4531 [astro-ph.CO]].

[7] M. Ackermann et al., "Constraining dark matter models from a combined analysis of Milky Way satellites with the Fermi-LAT," Phys. Rev. Lett. 107, 241302 (2011) [arXiv:1108.3546 [astro-ph.HE]].

[8] A. Geringer-Sameth and S. M. Koushiappas, Phys. Rev. Lett. 107, 241303 (2011) [arXiv:1108.2914 [astro-ph.CO]].

[9] A. A. Abdo et al., "Constraints on Cosmological Dark Matter Annihilation from the Fermi-LAT Isotropic Diffuse Gamma-Ray Measurement," JCAP 1004, 014 (2010) [arXiv:1002.4415 [astro-ph.CO]].

[10] J. F. Navarro, C. S. Frenk and S. D. M. White, "A Universal density profile from hierarchical clustering," Astrophys. J. 490, 493 (1997) [astro-ph/9611107].

[11] A. Pinzke, C. Pfrommer, and L. Bergstrom, "Prospects of detecting gamma-ray emission from galaxy clusters: cosmic rays and dark matter annihilations," arXiv:1105.3240 [astro-ph.HE].

[12] L. Gao, C. S. Frenk, A. Jenkins, V. Springel, and S. D. M. White, "Where will supersymmetric dark matter first be seen?," arXiv:1107.1916 [astro-ph.CO].

[13] M. A. Sanchez-Conde, M. Cannoni, F. Zandanel, M. E. Gomez and F. Prada, "Dark matter searches with Cherenkov telescopes: nearby dwarf galaxies or local galaxy clusters?," arXiv:1104.3530 [astro-ph.HE].

[14] A. M. Green, S. Hofmann and D. J. Schwarz, "The power spectrum of SUSY - CDM on sub-galactic scales," Mon. Not. Roy. Astron. Soc. 353, L23 (2004) [astro-ph/0309621].

[15] A. M. Green, S. Hofmann and D. J. Schwarz, "The First wimpy halos," JCAP 0508, 003 (2005) [arXiv:astro-ph/0503387 [astro-ph]]. 
[16] J. Diemand, B. Moore and J. Stadel, "Earth-mass dark-matter haloes as the first structures in the early Universe," Nature 433, 389 (2005) [astro-ph/0501589].

[17] J. Diemand, M. Kuhlen and P. Madau, "Early supersymmetric cold dark matter substructure," Astrophys. J. 649, 1 (2006) [astro-ph/0603250].

[18] S. Profumo, K. Sigurdson and M. Kamionkowski, "What mass are the smallest protohalos?," Phys. Rev. Lett. 97, 031301 (2006) [astro-ph/0603373].

[19] X. Huang, G. Vertongen and C. Weniger, "Probing Dark Matter Decay and Annihilation with Fermi LAT Observations of Nearby Galaxy Clusters," arXiv:1110.1529 [hep-ph].

[20] G. R. Blumenthal, S. M. Faber, R. Flores and J. R. Primack, "Contraction of Dark Matter Galactic Halos Due to Baryonic Infall," Astrophys. J. 301, 27 (1986).

[21] B. S. Ryden and J. E. Gunn, "Galaxy Formation by Gravitational Collapse," Astrophys. J. 318, 15 (1987).

[22] O. Y. Gnedin, A. V. Kravtsov, A. A. Klypin and D. Nagai, "Response of dark matter halos to condensation of baryons: Cosmological simulations and improved adiabatic contraction model," Astrophys. J. 616, 16 (2004) [astro-ph/0406247].

[23] O. Y. Gnedin, D. Ceverino, N. Y. Gnedin, A. A. Klypin, A. V. Kravtsov, R. Levine, D. Nagai and G. Yepes, "Halo Contraction Effect in Hydrodynamic Simulations of Galaxy Formation," arXiv:1108.5736 [astro-ph.CO].

[24] F. Prada, A. Klypin, J. Flix Molina, M. Martinez and E. Simonneau, "Dark Matter Annihilation in the Milky Way Galaxy: Effects of Baryonic Compression," Phys. Rev. Lett. 93, 241301 (2004) [astro-ph/0401512].

[25] M. Gustafsson, M. Fairbairn and J. Sommer-Larsen, "Baryonic Pinching of Galactic Dark Matter Haloes," Phys. Rev. D 74, 123522 (2006) [astro-ph/0608634].

[26] D. Merritt, A. W. Graham, B. Moore, J. Diemand and B. Terzic, "Empirical models for Dark Matter Halos. I. Nonparametric Construction of Density Profiles and Comparison with Parametric Models," Astron. J. 132, 2685 (2006) [astro-ph/0509417].

[27] J. F. Navarro, A. Ludlow, V. Springel, J. Wang, M. Vogelsberger, S. D. M. White, A. Jenkins and C. S. Frenk et al., "The Diversity and Similarity of Cold Dark Matter Halos," arXiv:0810.1522 [astro-ph].

[28] G. L. Bryan and M. L. Norman, "Statistical properties of x-ray clusters: Analytic and numerical comparisons," Astrophys. J. 495, 80 (1998) [astro-ph/9710107].

[29] A. R. Duffy, J. Schaye, S. T. Kay and C. Dalla Vecchia, "Dark matter halo concentrations in the Wilkinson Microwave Anisotropy Probe year 5 cosmology," Mon. Not. Roy. Astron. Soc. 390, L64 (2008) [arXiv:0804.2486 [astro-ph]].

[30] A. F. Neto, L. Gao, P. Bett, S. Cole, J. F. Navarro, C. S. Frenk, S. D. M. White and V. Springel et al., "The statistics of lambda CDM Halo Concentrations," Mon. Not. Roy. Astron. Soc. 381, 1450 (2007) [arXiv:0706.2919 [astro-ph]].

[31] L. Gao, J. F. Navarro, S. Cole, C. Frenk, S. D. M. White, V. Springel, A. Jenkins and A. F. Neto, "The redshift dependence of the structure of massive LCDM halos," arXiv:0711.0746 [astro-ph].

[32] D. H. Zhao, Y. P. Jing, H. J. Mo and G. Boerner, "Accurate universal models for the mass accretion histories and concentrations of dark matter halos," Astrophys. J. 707, 354 (2009) [arXiv:0811.0828 [astro-ph]].

[33] F. Prada, A. A. Klypin, A. J. Cuesta, J. E. Betancort-Rijo and J. Primack, "Halo concentrations in the standard LCDM cosmology," arXiv:1104.5130 [astro-ph.CO].

[34] W. Hu and A. V. Kravtsov, "Sample variance considerations for cluster surveys," Astrophys. J. 
584, 702 (2003) [astro-ph/0203169].

[35] J. A. Sellwood and S. S. McGaugh, "The Compression of dark matter halos by baryonic infall," Astrophys. J. 634, 70 (2005) [astro-ph/0507589].

[36] J. -H. Choi, Y. Lu, H. J. Mo and M. D. Weinberg, "Dark matter halo response to the disk growth," Mon. Not. Roy. Astron. Soc. 372, 1869 (2006) [astro-ph/0604587].

[37] P. Colin, O. Valenzuela and A. Klypin, "Bars and cold dark matter halos," Astrophys. J. 644, 687 (2006) [astro-ph/0506627].

[38] P. H. Johansson, T. Naab and J. P. Ostriker, "Gravitational Heating Helps Make Massive Galaxies Red and Dead," Astrophys. J. 697, L38 (2009) [arXiv:0903.2840 [astro-ph.CO]].

[39] M. G. Abadi, J. F. Navarro, M. Fardal, A. Babul and M. Steinmetz, "Galaxy-Induced Transformation of Dark Matter Halos," Mon. Not. Roy. Astron. Soc. 407, 435 (2010) [arXiv:0902.2477 [astro-ph.GA]].

[40] A. R. Duffy, J. Schaye, S. T. Kay, C. Dalla Vecchia, R. A. Battye and C. M. Booth, "Impact of baryon physics on dark matter structures: a detailed simulation study of halo density profiles," Mon. Not. Roy. Astron. Soc. 405, 2161 (2010) [arXiv:1001.3447 [astro-ph.CO]].

[41] P. B. Tissera, S. D. M. White, S. Pedrosa and C. Scannapieco, "Dark matter response to galaxy formation," Mon. Not. Roy. Astron. Soc. 406, 922 (2010) [arXiv:0911.2316 [astro-ph.CO]].

[42] A. Biviano and P. Salucci, "The radial profiles of the different mass components in galaxy clusters," Astron. Astrophys. 452, 75 (2006) [astro-ph/0511309].

[43] A. E. Schulz, R. Mandelbaum and N. Padmanabhan, "Testing adiabatic contraction with SDSS elliptical galaxies," Mon. Not. Roy. Astron. Soc. 408, 1463 (2010) [arXiv:0911.2260 [astro-ph.CO]].

[44] M. W. Auger, T. Treu, R. Gavazzi, A. S. Bolton, L. V. E. Koopmans and P. J. Marshall, "Dark Matter Contraction and the Stellar Content of Massive Early-type Galaxies: Disfavoring 'Light' Initial Mass Functions," Astrophys. J. 721, L163 (2010) [arXiv:1007.2409 [astro-ph.CO]].

[45] A. Sonnenfeld, T. Treu, R. Gavazzi, P. J. Marshall, M. W. Auger, S. H. Suyu, L. V. E. Koopmans and A. S. Bolton, "Evidence for dark matter contraction and a Salpeter IMF in a massive early-type galaxy," arXiv:1111.4215 [astro-ph.CO].

[46] T. H. Reiprich and H. Boehringer, "The Mass function of an X-ray flux-limited sample of galaxy clusters," Astrophys. J. 567, 716 (2002) [astro-ph/0111285].

[47] M. Ackermann et al., "GeV Gamma-ray Flux Upper Limits from Clusters of Galaxies," Astrophys. J. 717, L71 (2010) [arXiv:1006.0748 [astro-ph.HE]].

[48] A. Vikhlinin et al., "Chandra Cluster Cosmology Project II: Samples and X-ray Data Reduction," Astrophys. J. 692, 1033 (2009) [arXiv:0805.2207 [astro-ph]].

[49] S. Ando and D. Nagai, "Gamma-ray probe of cosmic-ray pressure in galaxy clusters and cosmological implications," Mon. Not. Roy. Astron. Soc. 385, 2243 (2008) [arXiv:0705.2588 [astro-ph]].

[50] Y. Chen, T. H. Reiprich, H. Bohringer, Y. Ikebe and Y. -Y. Zhang, "Statistics of X-ray observables for the cooling-core and non-cooling core galaxy clusters," Astron. Astrophys. 466, 805 (2007) [astro-ph/0702482 [ASTRO-PH]].

[51] E. Churazov, W. Forman, A. Vikhlinin, S. Tremaine, O. Gerhard and C. Jones, "Non-thermal pressure in M87 and NGC 1399 gas: X-ray vs. optical potential profiles," Mon. Not. Roy. Astron. Soc. 388, 1062 (2008) [arXiv:0711.4686 [astro-ph]].

[52] P. Das, O. Gerhard, E. Churazov and I. Zhuravleva, "Steepening mass profiles, dark matter and environment of X-ray bright elliptical galaxies," Mon. Not. Roy. Astron. Soc. 402, 1362 (2010) [arXiv:1007.5322 [astro-ph.CO]]. 
[53] T. R. Lauer et al., "The Centers of Early-Type Galaxies with HST.I.An Observational Survey," Astron. J. 110, 2622 (1995).

[54] T. R. Lauer et al., "The Centers of early-type galaxies with HST. 5. New WFPC2 photometry," Astron. J. 129, 2138 (2005) [astro-ph/0412040].

[55] T. R. Lauer et al., "The Centers of Early-Type Galaxies with HST. 6. Bimodal Central Surface Brightness Profiles," Astrophys. J. 664, 226 (2007) [astro-ph/0609762].

[56] J. Binney and S. Tremaine, "Galactic Dynamics," Princeton University Press, second edition (2008).

[57] R. P. Saglia, A. Kronawitter, O. Gerhard and R. Bender, "The Orbital Structure and Potential of NGC 1399," Astron. J. 119, 153 (2000).

[58] A. Kronawitter, R. P. Saglia, O. Gerhard and R. Bender, "Orbital structure and mass distribution in elliptical galaxies," Astron. Astrophys. Suppl. Ser. 144, 53 (2000).

[59] N. E. B. Killeen and G. V. Bicknell, "The mass distribution of NGC 1399 from optical and X-ray surface photometry," Astrophys. J. 325, 165 (1988).

[60] C. Jones, C. Stern, W. Forman, J. Breen, L. David, W. Tucker and M. Franx, "X-Ray Emission from the Fornax Cluster," Astrophys. J. 482, 143 (1997).

[61] M. Paolillo, G. Fabbiano, G. Peres and D. -W. Kim, "Deep ROSAT-HRI observations of the NGC 1399 / NGC 1404 region: Morphology and structure of the x-ray halo," Astrophys. J. 565, 883 (2002) [astro-ph/0109342].

[62] A. A. Abdo et al., "Fermi Large Area Telescope Second Source Catalog," arXiv:1108.1435 [astro-ph.HE].

[63] R. Trotta, "Bayes in the sky: Bayesian inference and model selection in cosmology," Contemp. Phys. 49, 71 (2008) [arXiv:0803.4089 [astro-ph]]. 